\title{
INTERLABORATORY PROGRAMS FOR RUBBER
}

\author{
ANALYSES NO. 35 \\ JANUARY - MARCH 1978
}
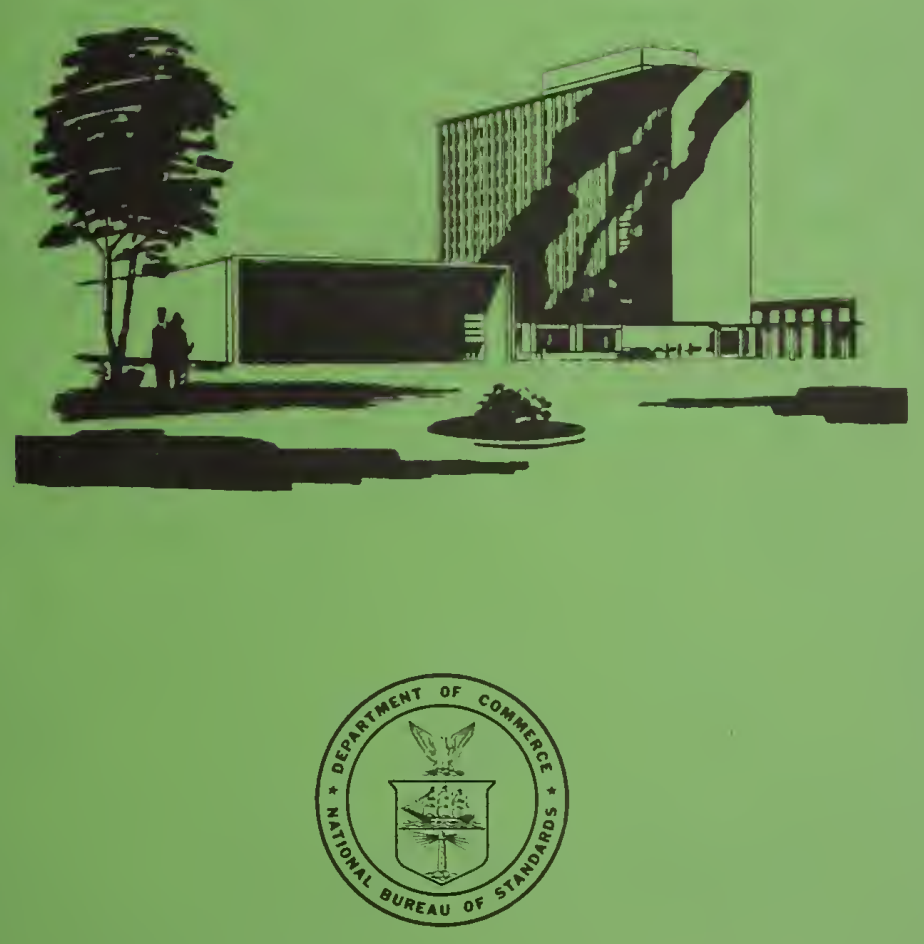

U. S. DEPARTMENT OF COMMERCE NATIONAL BUREAU OF STANDARDS 


\section{NBS COLLABORATIVE REFERENCE PROGRAMS}

TAPPI Paper and Board (6 times per year)

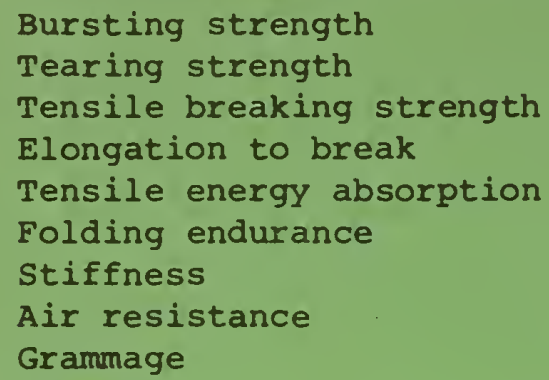

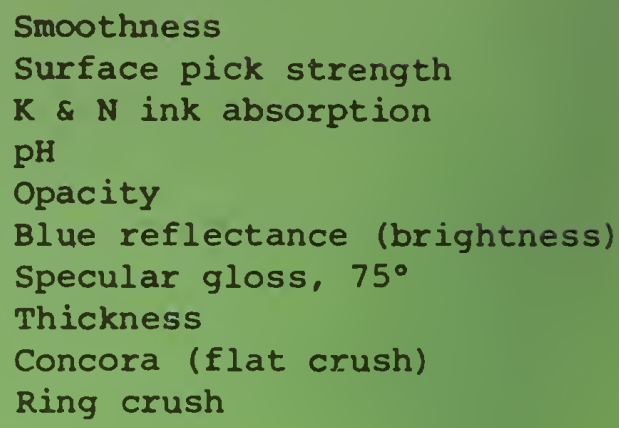

FKBG-API Containerboard (48 times per year)

Mullen burst of linerboard

Concora test of medium

MCCA Color and Appearance ( 4 times per year)

Gloss at $60^{\circ}$

Color and color difference

Retroreflectivity

Rubber (4 times per year)

Tensile strength, ultimate elongation and tensile stress Hardness

Mooney viscosity

Vulcanization properties

ASTM Textiles ( 3 times per year)

Flammability (FF3-71 and FF5-74)

ASTM Cement (2 times per year)

Chemical ( 11 chemical components)

Physical ( 8 characteristics)

\section{AASHTO Bituminous}

Asphalt cement ( 2 times per year) Cutbacks (once a year) 


\title{
INTERLABORATORY PROGRAMS FOR RUBBER
}

\author{
Analyses No. 35 \\ January - March 1978
}

\author{
J. Horlick \\ Office of Testing Laboratory Evaluation Technology \\ Office of Engineering Standards \\ National Engineering Laboratory \\ J. F. Stevenson, T. L. Cummings, Research Associates, \\ Collaborative Testing Services, Inc. \\ G. W. Bullman \\ Polymers Science and Standards Division \\ Center for Materials Research \\ National Measurement Laboratory
}

\section{U. S. DEPARTMENT OF COMMERCE National Bureau of Standards}




\section{INTRODUCTION}

This report summarizes the test results for the first quarter of 1978 . The tests cover the four areas in the NBS Collaborative Reference Programs for Rubber: Tensile Properties, Hardness, Mooney Viscosity, and Vulcanization Properties.

For each of the four areas, there is a set of summary tables followed by a table of data and analysis by laboratory and a graphical presentation of the data and analysis. Where applicable, the tables of data have the English and Metric expressions side-by-side. Additional details are given in the section "Key to Tables and Graphs."

If there are questions or comments on the notes, the analyses, or the reports in general, contact Edwin B. Randall, Jr., Jeffrey Horlick, or Jeffrey Stevenson, (301) 921-2946.
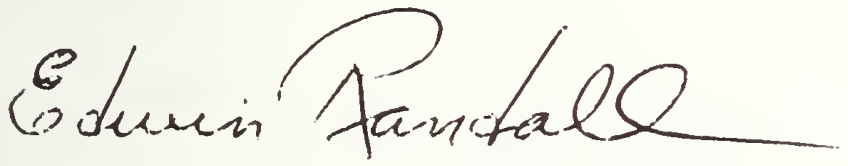

Edwin B. Randall, Jr., Administrator NBS Collaborative Reference Programs

Office of Testing Laboratory Evaluation Technology

June 12,1978 
TABLE OF CONTENTS

Page

Introduction

Table of Contents

Key to Tables and Graphs

Program 1: Tensile strength, ultimate elongation and stress at $300 \%$ elongation

Program 2: Hardness

Program 4: Mooney Viscosity

Program 5: Vulcanization characteristics determined with oscillating-disk cure meter 
LAB CODE Confidential laboratory identification number known only to the participant and the Collaborative Reference Program staff.

F

A flag identifying results that are extreme in comparison with the other results.

$\mathrm{X}$ - The plotted point for the indicated laboratory lies outside of the $99 \%$ error ellipse (not shown); ie, assuming normal distribution, $99 \%$ of laboratories similar to those participating in the program will be represented by points lying within the $99 \%$ ellipse.

- The plotted point for the indicated laboratory lies outside of the $95 \%$ error ellipse shown on graphs, but inside the $99 \%$ ellipse.

MEAN

$\% \mathrm{DEV}$

The arithmetic average of the two median values for the two sheets or samples of the same material.

The deviation or difference of the laboratory MEAN from the GR. MEAN (see below), expressed as a percent of the GR. MEAN .

REL SDR The ratio of the SDR (standard deviation of replicate measurements within a laboratory) to the AVER SDR (see below). Extreme values, ie, values that are likely to occur by chance less than one time in a hundred as determined by the chisquare test, are marked with an "X".

VAR CODE A code number designating a particular test instrument, set of environmental conditons, procedure, unit used, or other variation. The code "01" designates the instrument, conditions and procedure specified at the top of the page either explicitly or in the cited ASTM Standard, and the unit of test shown at the top of the first column of data. A ' + ' in front of the VAR CODE indicates that the data has been excluded from the grand means due to a non-standard variation of the possibilities mentioned above, or the data is extreme.

GR MEAN The arithmetic average (grand mean) of all the laboratory MEAN values, excluding those flagged (F) with an "X".

SD MEANS The standard deviation among the laboratory MEAN values included in the GR. MEAN. 
AVER SDR The arithmetic average of all the standard deviations of within laboratory replication, excluding those excluded from the GR. MEAN and excluding any additional ones for which the REL SDR has been flagged.

\section{GRAPH}

For each laboratory the MEAN for the second material is plotted against the MEAN for the first material, with each point representing a laboratory. The horizontal and vertical lines are the GR. MEAN values. The dashed line is drawn at $45^{\circ}$. The solid sloping line, which may or may not lie close to the $45^{\circ}$ line, is the major axis of the ellipse. The ellipse is drawn so that, on the average, it will include $95 \%$ of the points representing the laboratories. The plotted symbols $\mathrm{X}$ and $*$ used to represent results falling outside the ellipse are explained under " $F$ " above. Laboratories inside the ellipse (no flag in the F column) are plotted as an 0.

The graph is plotted with an ellipse when there are 20 or more laboratories in the analysis. When there are 10 through 19 laboratories in the analysis, the graph is plotted but the ellipse is omitted. When there are fewer than 10 laboratories retained in the Grand Mean the graph is not plotted.

For development of the theory, see the paper by J. Mandel and T.W. Lashof, Interpretation and Generalization of Youden's Two-Sample Diagram, J. of Quality Technology, Vol. 6, pp 22-36, Jan. 1974.

\section{SUMMARY OF ANALYSES}

LABS INCL Number of laboratories included in the GR. MEANs.

LABS OMIT Number of laboratories reporting data but excluded from the GR. MEANs.

STANDARD DEVIATIONS

LABS Same as the SD MEANs (see above)

SHEETS Standard deviation between the two sheets or samples of the same material.

REPL Same as AVER SDR (see above)

\section{PRECISION OF METHODS}

REPL CRP The number of replicate measurements per sheet or sample, as specified in the Collaborative Reference Program.

REPL ASTM The number of replicate measurements specified for a test result in the designated ASTM Standard. 
REPEAT The repeatability, a measure of the within laboratory precision, i.e., of the ability of the test technician to repeat his test result: two test results obtained by the same technician on the same homogeneous sample of material may be expected $95 \%$ of the time to agree within the repeatability.

REPROD The reproducibility, a measure of the between laboratory precision: two test results obtained in different laboratories may be expected $95 \%$ of the time to agree within the reproducibility.

ABSOLUTE Values of REPEAT and REPROD expressed in the units of measurement.

PERCENT Values of REPEAT and REPROD expressed as a percent of the GR. MEANs . 
NOTES

Materiala A81 and A82 were aheets of the same vulcanized rubber.

Similarly, materlals $\mathrm{A} 83$ and $\mathrm{A} 84$ were alike.

V200 results were obtained at NBS using an electronic tester, v100 results were obtained at NBS using an pendulum tester.

AII participants used Die C in ASTM D412 with the following exceptions:

$$
\begin{aligned}
& \text { V120 used ASTM Die B } \\
& \text { V126 used Die } 2 \text { in BS } 903 \\
& \text { V208 did not specify a Die } \\
& \text { V213 used ASTM Die D }
\end{aligned}
$$

Electronic testers were used by $39(64 \%)$ of the 61 participants; pendulum testers were used by 19 particlpants; 3 participants did not speclfy elther type. Elongation measurements were made by automatic devices by $22(36 \%)$ particlpants and manually by the rest. There were

\begin{tabular}{|c|c|c|c|c|c|c|c|c|c|c|c|}
\hline \multirow[b]{2}{*}{ PRAPERTY } & \multirow[b]{2}{*}{ NATERIAL } & \multirow{2}{*}{$\begin{array}{l}\text { LABS } \\
\text { INCL }\end{array}$} & \multirow{2}{*}{$\begin{array}{l}\text { LABS } \\
\text { GMIT }\end{array}$} & \multirow[b]{2}{*}{ GR, MEAN } & \multicolumn{3}{|c|}{ STD DEVIATIONS } & \multirow[b]{2}{*}{ UNITS } & & & \\
\hline & & & & & LABS & SHEETS & REPL & & & & \\
\hline TSNSILE & $A B 1-A B 2$ & 60 & 1 & 2657 . & 124 & 59. & 72. & PEONDS & PER & SOUARB & I NCH \\
\hline STRENGTH & $183-184$ & 60 & 1 & 3725. & 242. & 155. & 286 & PACNDS & PER & SQUARE & I NCH \\
\hline TENSILE & $181-182$ & 60 & 1 & 18.32 & .85 & .41 & .50 & MEGAPAS & CALS & & \\
\hline STRENGTH & 1830184 & 60 & 1 & 25.69 & 1.67 & 1.13 & 1.97 & SEGAPAS & CALS & & \\
\hline ULTINATE & $181 \cdot 182$ & 58 & 3 & 621. & 22. & 10. & 17. & PERCENT & & & \\
\hline LENGATIGN & 1830184 & 58 & 3 & 681. & 25. & 13. & 32. & PERCENT & & & \\
\hline STRE SS AT & $181=482$ & 59 & 2 & 1148. & 62. & 16. & 26. & PGENDS & PER & SQUARB & INC \\
\hline LENOATION & $A 83-A 84$ & 59 & 2 & 1013. & 61. & 20. & 30. & PAONDS & PER & SOOARE & I NC \\
\hline STRESS AT & $181-182$ & 59 & 2 & 7.916 & .429 & .121 & .178 & MESAPAS & CALS & & \\
\hline LONGATIGN & 1830.484 & 59 & 2 & 6.989 & .419 & .135 & .210 & MEGAPAS & CALS & & \\
\hline
\end{tabular}
$9(15 \%)$ reported relative humidities above $55 \%$ and $27(44 \%)$ reported relative humidities below $45 \%$.

SOKMARY GP ANALYSES

PRECISI GN GF VETHEDS

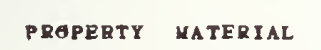

REPL RPPL
CRP ASTY

GR V VAN

A BSALUTB

PEPEAT REPRED

UN I TS

PERCENT

REPEAT REPRED

TBNSILE $\quad 81-\triangle 82$

STRENGTH AB3-184

$\begin{array}{ll}5 & 5 \\ 5 & 5\end{array}$

TENSILB AB1-A82

STRENGTH AB30A84

OLTIMATE ABI-AB

BLGNGATIGN A83-A84

STRESS AT A91-AB2

300\$ BLENGATIEN AB30ABA

STRESS $\triangle T$ TBI-AB2

$300 \%$ BLENGATIGN ABZ०AB4

$\begin{array}{rrrrrr}2657 . & 200 . & 343 . & \text { PSI } & 7.5 & 12.9 \\ 3725 . & 792 . & 671 . & \text { PSI } & 21.3 & 18.0 \\ 18.32 & 1.38 & 2.36 & \text { MESAPA } & 7.5 & 12.9 \\ 25.69 & 5.46 & 4.62 & \text { MEGAPA } & 21.3 & 18.0 \\ & & & & & \\ 621 . & 47 . & 61 . & \$ & 7.6 & 9.8 \\ 681 . & 88 . & 69 . & \$ & 13.0 & 10.2 \\ & & & & & \\ 1148 . & 71 . & 172 . & \text { PSI } & 6.2 & 15.0 \\ 1013 . & 84 . & 168 . & \text { PSI } & 8.3 & 16.5 \\ 7.916 & .493 & 1.187 & \text { MEGAPA } & 6.2 & 15.0 \\ 6.989 & .582 & 1.160 & \text { MEGAPA } & 8.3 & 16.6\end{array}$


YATERIAL $A 810 \triangle 82$ COMMERCIAL TIRE TREAD
LA8 MBAN MEAN CODE F PSI MEGAPA DEV SDR v0076 2740. $18.90 \quad 3.1 \quad 1.04$ vo 078 VOOB1 V0084

v0085

$\mathrm{Y} 0087$

บ0 08

vo 092

v0095

vo096

VOL 00

Vo1 \& \&

v 0217

v0120

vol $2 z$

V0123

V01 26

VOะ 28

VOI 42

VO1 $44 \mathrm{~A}$

VO1448

vo 145

V0148

Vol 49

v0150

vo151

vol 52

vol 53

Vole 4

v0156

V0158

vol 150

vos 60

vo1 66

vo168

v0163

VO1 16

Vo177

v0178

vo1 84

V0190

V0199

vo 200

VO2OE

VO20

VOลO

Vo 213

VOE 14

Vo 220

VOE 23

V0 224

V0232

vo 233

vo 235

v0238

Vo 243

VO 244

Vo $245 A$

v02458.

vo 250

$\begin{array}{llll}2695 . & 18.59 & 1.4 & .59\end{array}$

2611. $18.00 \quad-1.7 \quad .71$

2590. $17.86 \quad-2.5 \quad 1.11$

$2420 . \quad 16.69 \quad-8.9 \quad .76$

2620. $18.07 \quad-1.4 \quad 1.32$

$2700.18 .62 \quad 1.6 \quad 1.00$

2612. $18.01 \quad-1.7 \quad .83$

2690. 18.55 $1.2 \quad 1.21$

2770. 19.10 $4.3 \quad 1.04$

$\begin{array}{lllll}2645 . & 18.24 & 0.5 & 1.24\end{array}$

$\begin{array}{llll}2657 . & 18.32 & .0 & .85\end{array}$

2405. $16.59 \quad-9.5 \quad 1.01$

2735. 18.86 E.9 1.05

2821. $19.46 \quad 6.2 \quad .94$

2665. 18.38 .3.89

2808. $19.37 \quad 5.7 \quad 1.52$

2915. $20.10 \quad 9.7 \quad .56$

2655 .

2517.

¿ 730 .

2705.

2604 .

2850 .

2555.

¿520.

18.31

$17.36-5.3 \quad .93$

$\begin{array}{rrr}18.82 & 2.7 & .93\end{array}$

$18.66 \quad 1.8 \quad .90$

$\begin{array}{lll}17.96 & -2.0 \quad .90\end{array}$

$\begin{array}{lll}19.66 & 7.3 & 1.80\end{array}$

$\begin{array}{lll}17.62 & -3.8 & 1.36\end{array}$

$\begin{array}{lll}17.38 & -5.2 & .72\end{array}$

2765. $19.07 \quad 4.1 \quad 1.20$

$\begin{array}{lllll}2777 . & 19.15 & 4.5 & 1.15\end{array}$

2525. $17.41=5.0 \quad 1.27$

E64C. $18.21 \quad 0.6 \quad .82$

1713. $18.7: \quad 2.1 \quad .36$

2834.

2451 .

टC 45 .

2760

2715.

$18.24 \quad 0.5 \quad .75$

2630.

$264 \mathrm{C}$.

$18.14 \quad-1.0 \quad .51$

$18.21 \quad=.6 \quad 1.01$

$\begin{array}{lll}18.60 & 1.5 & 1.67\end{array}$

$2704.18 .65 \quad 1.8 \quad 1.75$

2550 .

$\begin{array}{rrr}17.59 & -4.0 \quad .86\end{array}$

$20.34 \quad 11.0 \quad 1.18$

$\begin{array}{lll}19.51 & 6.4 & .72\end{array}$

$\begin{array}{lll}18.79 & 2.6 & 1.28\end{array}$

2725 .

19.31

$5.4 \quad .72$

2725.

$\overline{2605 .}$

$18.79 \quad 2.6 \quad 1.56$

$18.21 \quad-.6 \quad 1.05$

$2 \epsilon 4 \mathrm{C}$.

2708 .

$18.68 \quad 1.9 \quad .56$

2637.

$18.19 \quad-.7 \quad .87$

$\begin{array}{lll}17.63 & -3.8 & 1.01\end{array}$

$17.51=.4 \quad 1.01$

\section{YATERIAL AB3-A84}

SBR
MEAN MEAN

PSI MEGAPA DBV SOR CEDE

3640. $25.10 \quad-2.3 \quad 1.40 \quad 01$

358c. $24.69-3.9 \quad 1.48 \quad 01$

$\begin{array}{lllll}4025 . & 27.76 & 8.0 & .87 & 01\end{array}$

$\begin{array}{lllll}3650 . & 25.17 & -2.0 & .57 & 01\end{array}$

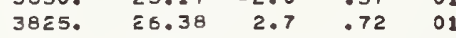

$19.14 \quad 4.4 \quad 2.14 x$

$19.54 \quad 6.7 \quad 2.71 x$

$16.90 \quad-7.7 \quad .56$

$\begin{array}{lll}19.03 & 3.9 & 1.29 \\ 18.72 & 2.2 & 2.36 x\end{array}$

$\begin{array}{lll}18.62 & 1.6 & 1.16\end{array}$

$17.97=2.0 \quad 2.36 x$

$\begin{array}{lll}17.76 & =3.1 & .55\end{array}$

$16.77-8.5 \quad 1.99 x$

$15.84=13.5 \quad .98$

$\begin{array}{llll}2687 . & 18.53 & 1.1 & 1.40\end{array}$

2657.

124.

$18.32 \cdot$ GR. MEAN -

.85 - SD MEANS -

.50 - AVER SDR .

PSI

MEOAPA - AVER SDR -

\section{7 .}

3505 .

3550 .

3540.

3950 .

3432 .

3865.

3990 .

3990 .

3268.

3600 .

3855.

4097.

3615 .

3782 .

25. 36

$24.17=5.9 \quad 1.45$

$24.48 \quad 04.7 \quad .96 \quad 01$

$24.41 \quad-5.0 \quad 1.32 \quad 01$

$23.67 \quad-7.9 \quad 1.01 \quad 01$

$\begin{array}{llll}26.66 & 3.7 & .90 & 01\end{array}$

$27.52 \quad 7.1 \quad .64 \quad 01$

$22.54012 .3 \quad .95 \quad 01$

$24.83 \quad-3.4 \quad 1.71 \quad 01$

$\begin{array}{llll}26.59 & 3.5 \quad .34 & 01\end{array}$

$28.26 \quad 10.0 \quad .45 \quad 20$

$24.93=3.0 \quad .82 \quad 01$

$\begin{array}{llll}26.09 & 1.5 & 1.08 & 01\end{array}$

3915. $27.00 \quad 5.1 \quad 1.21 \quad 01$

$\begin{array}{lllll}3700 . & 25.52 & \ldots .7 & 1.28 & 01\end{array}$

3553. $24.51 \quad-4.6 \quad 1.17 \quad 01$

$4225.25 .14 \quad 13.4 \quad 1.19$ O

$\begin{array}{lllll}3742 . & 25.81 & .4 & 1.31 & 20\end{array}$

3840. $26.48 \quad 3.1 \quad .9401$

4135. $28.52 \quad 11.0 \quad 1.17 \quad 01$

3820. 26.5a $2.5 \quad .83 \quad 01$

$\begin{array}{lllll}3475 . & 23.97 & -6.7 & 1.12 & 01\end{array}$

3900. $2 \epsilon .90 \quad 4.7 \quad .87 \quad 01$

$\begin{array}{lllll}3655 . & 25.21 & -1.9 & 1.66 & 20\end{array}$

3555. $\quad 24.59 \quad 04.3 \quad .76 \quad 01$

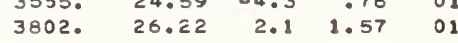

$3710.25 .590 .4 \quad 1.01 \quad 01$

3545. $24.46 \quad-4.8 \quad .90 \quad 20$

$\begin{array}{llrrr}3810 . & 28.28 & 2.3 & .84 & 01\end{array}$

$\begin{array}{lllll}3885 . & 26.79 & 4.3 & .83 & 01\end{array}$

3931. $27.11 \quad 5.5 \quad .63 \quad 01$

3778. $26.06 \quad 1.4 \quad .62 \quad 01$

3546. $24.46 \quad-4.8 \quad .73 \quad 01$

$4001.27 .60 \quad 7.4 \quad .7 \epsilon \quad$ CI

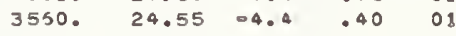

4310. $29.72 \quad 15.7 \quad .82 \quad 01$

$\begin{array}{lllll}3989 . & 27.51 & 7.1 & .91 & 20\end{array}$

$3673 . \quad 25.35 \quad 01.4 \quad .67 \quad 01$

3823. $26.37 \quad 2.5 \quad 1.23 \quad 20$

3720. $25.66 \quad 0.1 \quad 1.29 \quad 01$

$3757.25 .91 \quad .9 \quad 1.01 \quad 01$

$\begin{array}{lllll}3780 . & 26.07 & 1.5 & .73 & 01\end{array}$

$\begin{array}{lllll}3893 . & 26.85 & 4.5 & .98 & 01\end{array}$

$\begin{array}{lllll}3805 . & 26.24 & 2.1 & .97 & 01\end{array}$

$\begin{array}{lllll}3925 . & 27.07 & 5.4 & 1.77 & 01\end{array}$

3536. $24.39 \quad-5.1 \quad 1.08 \quad 01$

$\begin{array}{rrrrr}3513 . & 24.23-5.7 & .70 & 21 \\ 3100 . & 21.38-16.8 & .78 & 01\end{array}$

3029. $20.89-18.7 \quad .71 \quad 01$

3400 .

$23.45 \quad-8.7 \quad 1.97 \times \quad 01$

3725. 25.69

242. 1.67

286. 1.97

PSI MEGAPA
3649. $25.17 \quad 02.1 \quad 1.49 \quad 01$
INSTRUYENT, UNIT, GR GTHER VARIATION

ORIGINAL IN YBGANETTENS PER SQ METER

ORIGINAL IN MEJANETTENS PER SO.NETER

ORIGINAL IN MEGANETTONS PER SO. METER

ORIGINAL IN MBGANEWTENS PER SP.MBTER

ORIGINAL IN MBJANBWTGNS PER SP. NETER

ORIGINAL IN MEGANEWTANS PER SO. NETER GRIGINAL IN MEGANERTENS PER SO, METER

orIGINAL IN KILGGRANS/SQ. CENTIMETFR

5 TEST DETERMINATIONS

60 LABERATERIBS IN GRAND NEANS

61 LA 3ERATERIBS RBPORTING 
TENSILE STRENGTH

MATERIAL A81-A82 2657. PSI MATERIAL 983-A84 3725. PSI

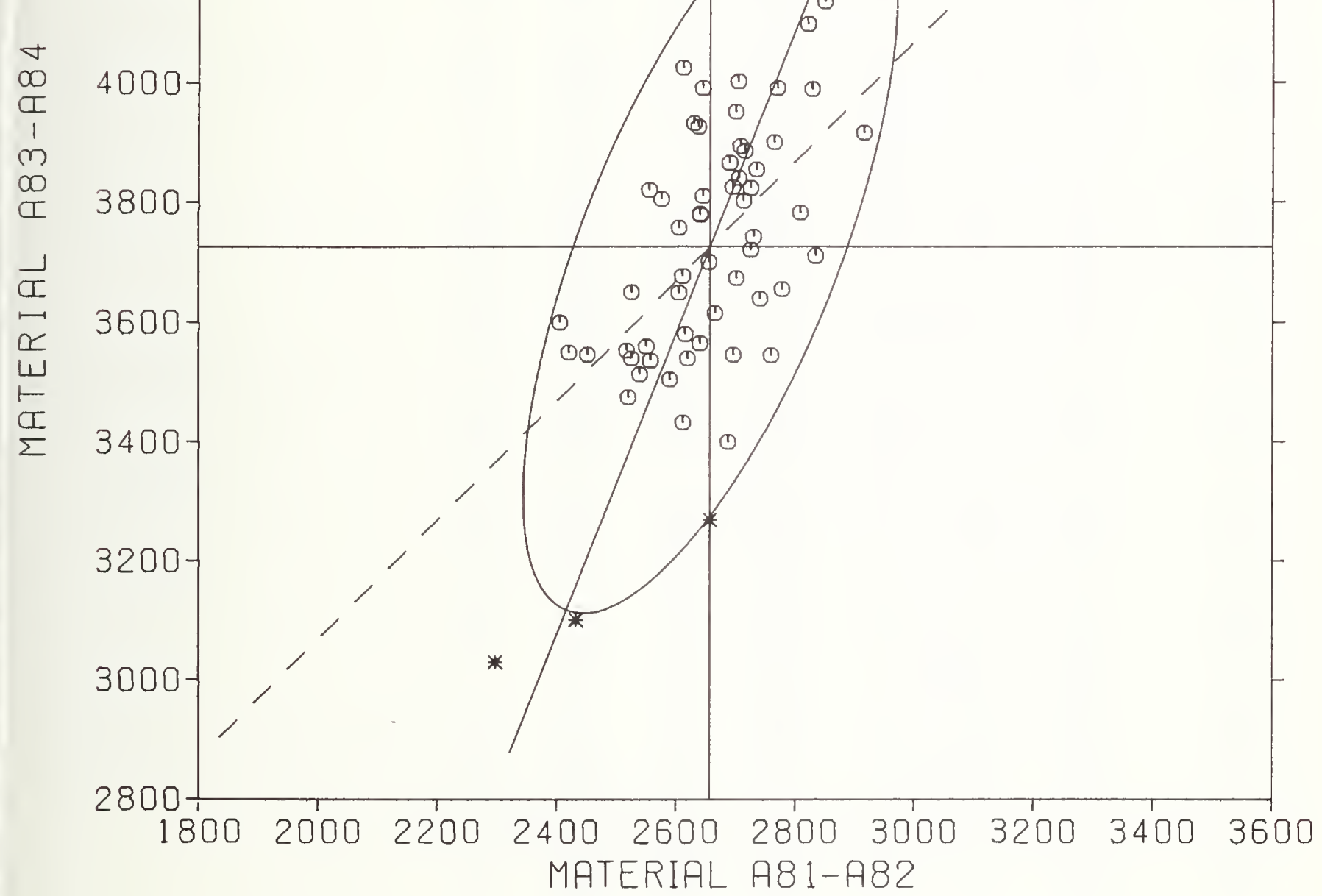


INTERLABORATERT PREGRAY ON EVALUATIEN GP RUBRER

ULTIMATE BLONGATION - PBRCBNT

MATBRIAL $\triangle 83-184$ SBR

COMMERCIAL TIRE TRB

LAB MEAN

$\begin{array}{lll}\text { LAB } & \text { MEAN DEL }\end{array}$

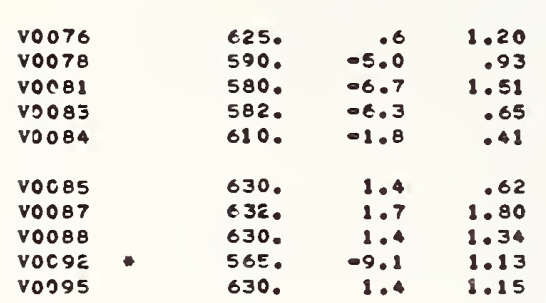

v0096

vo100

v0111

vo11 17

vol 20

$621 . \quad .0 \quad 1.27$

$615 . \quad-1.0 \quad .92$

$643 . \quad 3.5 \quad 1.28$

$635 . \quad 2.2 \quad .85$

Y01 22

vol 23

V0126

Yo1 28

VO141

V0144A

VC 1448

Vo145

VO148

vol 49

VO1 52

vol 52

vo1 53

vo1 54

vo1 56

v0158

vo159

vo1 60

vo166

vo168

vo1 69

V0176

v0177

vo178

VO1 84

vo1 90

v0199

V0200

v0206

v0207

VO 208

vo213

vo21.

Vo2 20

$\operatorname{voc} 23$

vo232

vo2 33

vo 235

Vo 238

V0243

v0244

Yoz $45 \mathrm{~A}$

Vo2 $45 B$

vo250 $x$
$640 . \quad 3.0 \quad 1.07$

625. $\quad .6 \quad 1.21$

$641 . \quad 3.2 \quad .81$

$610 . \quad-1.8 \quad 1.51$

635.2 .21 .29

$640.3 .0 \quad .75$

620.05

$645 . \quad 3.8 \quad .85$

$625 . \quad .6 \quad 1.13$

585. $-5.9 \quad .98$

625.

655.

61 .

$612 . \quad-1.4 \quad 1.46$

$595 . \quad-4.2 \quad .59$

$600 . \quad-3.4 \quad 1.50$

685. 10.21 .01

$615 . \quad-1.0 \quad 1.45$

$610 . \quad-1.8 \quad .89$

$620 . \quad-.2 \quad .26$

630.

585.

615 .

$\begin{array}{rc}1.4 & 2.41 x \\ -5.9 & .45 \\ -1.0 & .93\end{array}$

$660 . \quad 6.2 \quad .91$

628. $1.12 .40 x$

$$
\begin{array}{rrr}
620 . & -.2 & .63 \\
645 . & 3.8 & .57 \\
660 . & 6.2 & 2.10 x \\
615 . & -1.0 & 1.27 \\
640 . & 3.0 & 1.06
\end{array}
$$

615. $-1.0 \quad .60$

$600 . \quad-3.4 \quad .49$

$641 . \quad 3.2 \quad 1.22$

$640 . \quad 3.0 \quad 1.33$

$655 . \quad 5.4 .87$

\section{0.}

620.

615.

$\begin{array}{lll}600 . & -3.4 & .49 \\ 585 & -5.9 & .58\end{array}$

625.

625.

$610^{-1.6} \quad .72$

$615 . \quad-1.0 \quad 1.90$

630.11 .41 .00

665. $7.0 \quad .21$

621. 32 . MBAN -

22. SD UEANS

17. - AVBR SDR -

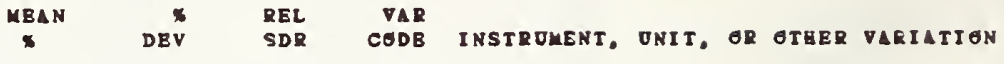

$\begin{array}{llll}650 . & -4.6 & 1.51 & 01 \\ 650 . & -4.6 & 1.81 & 01 \\ 660 . & -3.1 & .77 & 01 \\ 677 . & 0.6 & .73 & 01 \\ 680 . & 0.2 & .82 & 01\end{array}$

$710 . \quad 4.2 \quad 1.43 \quad 01$

$662 . \quad-2.9 \quad 1.59 \quad 01$

$6150-0.7 \quad 1.17 \quad 01$

695. 2.C 1.49

$650 . \quad-4.6 \quad 1.22 \quad 01$

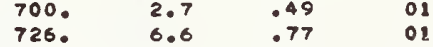

720.01 .75 o1

635. $-6.8 \quad 1.14 \quad 01$

$\begin{array}{llll}700 . & 2.7 & 1.89 & 01\end{array}$

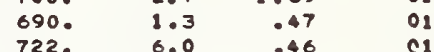

$675 . \quad 0.901010$

$670 . \quad-1.7 \quad .89 \quad 01$

350. $1.3 \quad 1.14 \quad 01$

$\begin{array}{llll}695 . & 2.0 & .81 & 01 \\ 705 . & 3.5 & 1.32 & 01\end{array}$

$\begin{array}{rrrr}705 . & 3.5 & 1.32 & 01 \\ 700 . & 2.7 & .55 & 01 \\ 662 . & -2.8 & .74 & 01\end{array}$

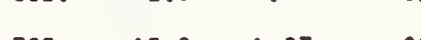

$\begin{array}{rrll}785 . & 15.2 & 1.27 & 01 \\ 735 . & 7.9 & 2.01 \times & 01\end{array}$

$685 . \quad .5 \quad 1.16 \quad 01$

$\begin{array}{lrrr}700 . & 2.7 & .78 & 01 \\ 655 . & -3.9 & 1.18 & 01\end{array}$

650. $-4.6 \quad .60 \quad 01$

$\begin{array}{llll}700 . & 2.7 & 1.71 & 01\end{array}$

$\begin{array}{llll}565 . & -2.4 & .76 & 01 \\ 650 . & -4.6 & .63 & 01\end{array}$

$\begin{array}{llll}700 . & 2.7 & 1.76 & 01\end{array}$

$645 . \quad-5.3 \quad .83 \quad 01$

$660 . \quad-3.1 \quad 07 \quad 01$

$675 . \quad-.9 \quad 1.19 \quad 01$

$691 . \quad 1.4 \quad .76 \quad 01$

$\begin{array}{llrl}675 . & 0.9 & .87 & 01 \\ 700 . & 2.7 & .05 & 01 \\ 675 . & 0.9 & 1.07 & 01 \\ 700 . & 2.7 & .49 & 01 \\ 680 . & 0.2 & 1.13 & 01\end{array}$

680.1 .13 01

$\begin{array}{rrrr}760 . & 2.7 & .45 & 01 \\ 660 . & -3.1 & .78 & 01\end{array}$

$684 . \quad .4 \quad .79 \quad 01$

$715 . \quad 4.9 \quad 1.52 \quad 01$

680. $\quad .2 \quad 1.37 \quad 01$

$\begin{array}{rrrr}735 . & 7.9 & 1.04 & 01 \\ 690 . & 1.3 & .90 & 01\end{array}$

$\begin{array}{llll}690 . & 1.3 & .90 & 01 \\ 660 . & -3.1 & .82 & 01 \\ 655 & -3.9 & .58 & 01\end{array}$

655.

$\begin{array}{rrrr}688 . & .9 & 1.63 & 01 \\ 685 . & .5 & .88 & 01\end{array}$

$680 . \quad-.2 \quad .70$

670. $-1.7 \quad 1.13 \quad 01$

705. $3.5 \quad 1.08 \quad 01$

635. $-6.8 \quad 1.99 \times \quad 01$

681.

25.

32 .
5 TEST DBTERUINATI GNS

58 LABORATERIBS IN ORAND MEANS 6) LABORATORIBS RBPORTINO 


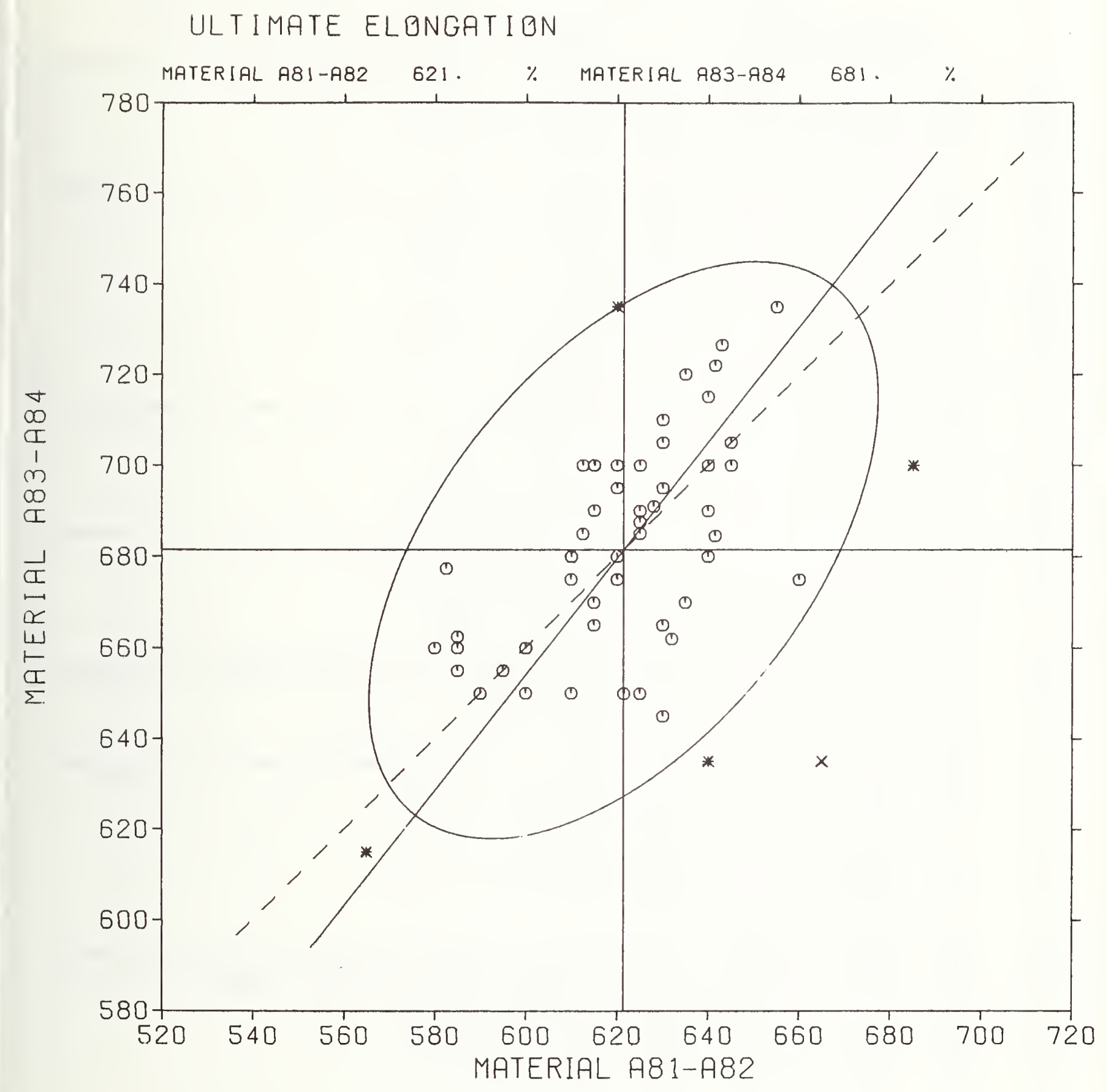


INTERLABGRATERY PREGRAM ON EVALUATION GF RUBBER

STRESS AT 300\% BLONGATIEN - POUNDS PER SQUARB INCB

MATERIAL AB BOAB4

MATEL $A 810 A 82$

\begin{tabular}{|c|c|c|c|c|c|c|c|c|c|c|c|c|c|}
\hline & & Con & ERCIAL & TIRE TRE & EAD & & SBR & 等 & & & & & \\
\hline LA 8 & & MEAN & MEA $\mathrm{N}$ & $x$ & REL & MEAN & NEAN & 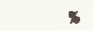 & RBL & $\mathbf{V}_{\mathbf{A} \mathbf{R}}$ & & & \\
\hline CODE & $\mathbf{F}$ & PSI & MEGAPA & DBV & SDR & PSI & MEGAPA & DEV & SDR & $\operatorname{CODE}$ & INSTRUMENT. & ONII, OR GTEER & VARIAIIEN \\
\hline vo07e & & 1175 & 8.103 & 2.4 & .67 & 1040 & 7.172 & 2.6 & .65 & 01 & & & \\
\hline v0078 & & 1179. & 8.131 & 2.7 & 1.10 & 1031 . & 7.114 & 1.8 & 1.10 & 01 & & & \\
\hline voo8: & & 1825. & 7.759 & $=2.0$ & .75 & 1062. & 7.328 & 4.8 & .83 & 01 & & & \\
\hline v0083 & & 1162. & 8.017 & $1 \cdot 3$ & .70 & 1050 & 7.241 & 3.6 & .85 & 01 & & & \\
\hline vo0 84 & & 1165. & 8.034 & $\therefore .5$ & .60 & 1025 & 7.069 & 1.1 & .70 & 01 & & & \\
\hline V0085 & & 1131. & 7.802 & -1.4 & .39 & 972. & 6.702 & $=4.1$ & .91 & 20 & ORIGINAL IN & MBOANEWTONS PER & SQ. METER \\
\hline vo0 87 & & 1155. & 7.966 & .5 & .90 & 1050 & 7.310 & 4.6 & .96 & 01 & & & \\
\hline v0088 & & 1070. & 7.379 & -5.8 & $2.06 x$ & 1030 & 7.103 & 1.6 & 1.69 & 01 & & & \\
\hline v0092 & & 1285 & 8.862 & 12.0 & 1.23 & 1135 & 7.828 & 12.0 & 1.05 & 01 & & & \\
\hline vo095 & & 1175 . & 8.103 & 2.4 & 1.13 & 1090 & 7.517 & 7.6 & .93 & 08 & & & \\
\hline v0096 & & 1158 & 7.990 & .9 & 1.09 & 1020 . & 7.038 & .7 & .83 & 01 & & & \\
\hline v0100 & & 1140. & 7.862 & 0.7 & .98 & 990. & 6.828 & -2.3 & 1.26 & 01 & & & \\
\hline v0111 & & 1165 & 8.034 & 1.5 & .67 & 1035 . & 7.138 & 2.1 & .66 & 01 & & & \\
\hline vo1 17 & & 1120 . & 7.724 & -2.4 & 1.38 & 965. & 6.655 & -4.8 & 1.61 & 01 & & & \\
\hline v0120 & & 1818 & 7.714 & -2.6 & .56 & 1036 & 7.148 & 2.3 & 1.49 & 01 & & & \\
\hline V0122 & $\mathbf{x}$ & 862. & 5.948 & .24 .9 & 1.25 & 825 & 5.690 & .18 .5 & 1.03 & 01 & & & \\
\hline V0123 & & 1195. & 8. 241 & 4.1 & .87 & 1050. & 7.241 & 3.6 & 1.01 & 01 & & & \\
\hline VO1 $\geq E$ & & 1849. & 7.927 & .1 & 1.00 & 575. & 6.727 & -3.8 & .74 & 20 & GRIGINAL IN & MEGANEETONS PER & SQ. MBTER \\
\hline V0128 & & $\$ 190$. & 8.207 & 3.7 & .55 & 1040. & 7.172 & 2.6 & 1.12 & 01 & & & \\
\hline VO 141 & & 1229 & 8.476 & 7.1 & .43 & 1037. & 7.152 & 2.3 & .49 & 01 & & & \\
\hline VO144A & & 1140. & 7.862 & .07 & 1.39 & 950. & 6.552 & -6.3 & .76 & 01 & & & \\
\hline vo144B & & 1140 & 7.862 & 0.7 & 1.72 & 960. & 6.621 & -5.3 & .85 & 01 & & & \\
\hline V0146 & $*$ & 979. & $6.75 \hat{2}$ & 014.7 & 1.16 & 910. & 6.276 & -10.2 & 1.16 & 09 & & & \\
\hline V0148 & & $\$ 200$. & 8.276 & 4.5 & .43 & 1087 & 7.500 & 7.3 & 1.08 & 01 & & & \\
\hline v0149 & & 1198. & 8.252 & 4.4 & .70 & 1053. & 7.262 & 3.9 & 1.20 & 20 & GRIGINAL IN & YESANETTONS PER & SQ. METER \\
\hline vo1 $5 \mathrm{C}$ & & 1125 & 7.759 & .2 .0 & .59 & 965. & 6.555 & -0.8 & .53 & 01 & & & \\
\hline Vo1 51 & & $1: 05$. & 7.621 & -3.7 & $2.34 x$ & 961. & 6.631 & $=5.1$ & $2.37 x$ & 01 & & & \\
\hline vo152 & & 1210 & 8.345 & 5.4 & .38 & $1 C 90$ & 7.517 & 7.6 & .69 & 01 & & & \\
\hline vo\& 53 & & $1:<0$. & 7.728 & $=2.4$ & 1.78 & 961. & 6.628 & $=5.2$ & 1.74 & 01 & & & \\
\hline vo 154 & & 1160 & 8.000 & $1 \cdot 1$ & .47 & 1035 & $7.1 \Xi 8$ & 2.1 & .81 & 01 & & & \\
\hline vo1 156 & & 1225. & 8.448 & 6.7 & 1.49 & 1065 . & 7.345 & 5.1 & 1.18 & 01 & & & \\
\hline vo 158 & & 1073. & 7.402 & -6.5 & .98 & 943. & 6.502 & $=7.0$ & .32 & 20 & ORIGINAL IN & MBZANBVTUNS PER & S). METER \\
\hline v0159 & & 1110. & 7.655 & $-3 \cdot 3$ & 1.00 & 1020 . & 7.034 & .6 & 1.17 & $C_{2}$ & & & \\
\hline VOI 160 & & 1175 . & 8.103 & 2.4 & .93 & 1070 & 7.379 & 5.6 & 1.13 & 01 & & & \\
\hline$v 0166$ & & 1148. & 7.921 & .1 & .78 & 973. & 6.710 & -4.0 & .59 & 01 & & & \\
\hline V0168 & & 1187 & 8.186 & 3.4 & 1.30 & 1013. & 6.990 & .0 & .90 & 01 & & & \\
\hline vo\& 59 & & 1132. & 7.802 & $=1.4$ & 1.13 & 1008 & 6.952 & 0.5 & 1.17 & 20 & ORIGINAL IN & MBGANE TONS PER & SQ.MBTER \\
\hline V0175 & & 1155. & 7.966 & .6 & 1.75 & 985. & 6.793 & -2.8 & 1.39 & 01 & & & \\
\hline Vo 177 & & 1065. & $7 \cdot 345$ & $=7.2$ & .74 & 940. & 6.483 & -7.2 & 1.20 & 01 & & & \\
\hline Vo $1>8$ & & 1135. & 7.828 & -1.1 & .74 & $\$ 85$ & 6.793 & -2.8 & .63 & 01 & & & \\
\hline VO 184 & & 1067. & 7.362 & $=7.0$ & .84 & 1025 & 7.069 & 1.1 & .47 & 01 & & & \\
\hline v0190 & & 1153. & 7.955 & .5 & 1.55 & 976. & 6.731 & -3.7 & 1.04 & 01 & & & \\
\hline V0199 & & 1123. & 7.748 & $=2.1$ & 1.77 & 1043 & 7.197 & 3.0 & 1.86 & 01 & & & \\
\hline $\mathrm{VO} 20 \mathrm{C}$ & & 1107. & 7.638 & -3.5 & .94 & \$ 85 & 6.707 & -2.8 & .54 & 01 & & & \\
\hline VOE 06 & & $111 \mathrm{C}$ & 7.655 & -3.3 & 1.37 & 1020 & 7.034 & .5 & 1.62 & 01 & & & \\
\hline vo 207 & - & 1300 & 8.966 & 13.3 & 1.81 & 1195. & 8.241 & 17.9 & 1.09 & 01 & & & \\
\hline Vo 208 & & 1226. & 8.452 & 6.8 & $2.37 x$ & 1139. & 7.852 & 12.3 & $2.11 \mathrm{x}$ & 20 & ORIGINAL IN & MBGANBWTONS PER & SQ. METER \\
\hline Vo 213 & & 1140 . & 7.866 &. .6 & 1.65 & 977. & 6.741 & -3.5 & 1.44 & 01 & & & \\
\hline VO 214 & & 1102 . & 7.502 & .4 .0 & .55 & 914. & 6.302 & -0.8 & 1.00 & 20 & ORIGINAL IN & MBGANETTENS PRR & SQ. METFR \\
\hline v0220 & & 1150. & 7.931 & $\cdot 2$ & .75 & 1000 & 6.897 & -1.3 & .70 & 01 & & & \\
\hline v0 $2 \approx 3$ & & 1220. & 8.414 & 6.3 & .43 & 1025 & 7.069 & 1.1 & .97 & 01 & & & \\
\hline vo224 & & 1180. & 8.138 & 2.8 & 1.43 & 1000 & 6.897 & -1.3 & 1.08 & 01 & & & \\
\hline vo 232 & & 1155 & 7.966 & .6 & 1.16 & 1002 . & 6.914 & -1.1 & .89 & 01 & & & \\
\hline vo 233 & & 1212 & 8.362 & 5.6 & 1.03 & 1067 & 7.359 & $5 \cdot 3$ & 1.10 & 01 & & & \\
\hline Vo 235 & & 1215 & 8.379 & 5.9 & .78 & 1110 & 7.655 & 9.5 & 1.10 & 01 & & & \\
\hline vo 238 & & 1112. & 7.672 & -3.1 & 1.07 & 975. & 6.724 & 03.8 & .63 & 01 & & & \\
\hline vo 243 & & 1013 & 6.986 & -11.7 & .56 & 889 . & 6.131 & 012.3 & .72 & 01 & & & \\
\hline vo 244 & & 1088 & 7.504 & -5.2 & 1.50 & 581. & 6.768 & -3.2 & 1.21 & 21 & ORIGINAL IN & KILJORAKS/SQ. CB & BNTIKETER \\
\hline V024 $5 A$ & * & 959. & 6.617 & -16.4 & 1.54 & 860 . & 5.934 & -15.1 & 1.35 & 01 & & & \\
\hline v02458 & $\mathbf{x}$ & $\varsigma 25$. & 6.379 & -19.4 & 1.04 & 718 & 4.955 & -29.1 & 1.75 & 01 & & & \\
\hline v0250 & & 1212 & 8.362 & 5.6 & .97 & $\$ 87$. & 5.810 & -2.6 & .82 & 01 & & & \\
\hline & & 1148 & 7.916 & - GR. & MEAN & 1013. & 6.989 & & & 5 & TBST DETERM & INATIONS & \\
\hline & & 62. & .429 & $=$ SD $\mathrm{M}$ & IEANS & 61. & .419 & & & 59 & LA BGRATERIE & S IN GRAND MEANS & \\
\hline & & $\begin{array}{l}26 . \\
\text { PSI }\end{array}$ & $\begin{array}{r}178 \\
\text { YEGAPA }\end{array}$ & $\begin{array}{rr}- & \Delta V E R \\
\text { - } & \text { UN }\end{array}$ & $\begin{array}{l}\text { SDR } \\
I T\end{array}$ & $\begin{array}{l}30 . \\
\text { PSI }\end{array}$ & $\begin{array}{r}.210 \\
\text { YEGAPA }\end{array}$ & & & 61 & LABGRATERIE & S REPORTING & \\
\hline
\end{tabular}


STRESS AT 300\% ELONGATION

MATERIAL A81-982 1148. PSI MATERIAL 983-984 1013. PSI

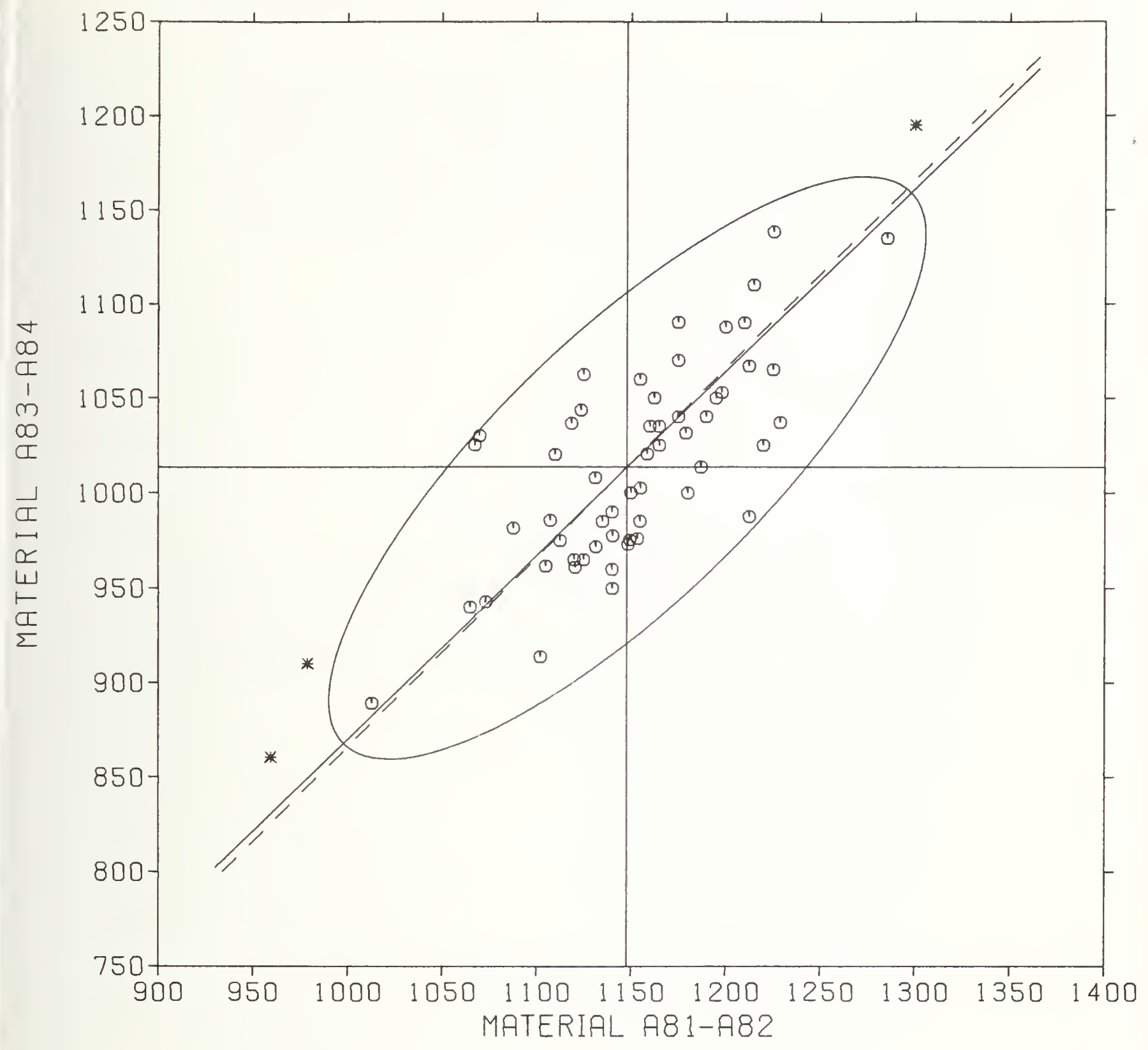


HARDNESS

NOTES

Materials $A 81$ and $A 82$ were sheets of the same vulcanized rubber. Similarly, materlals $\mathrm{A} 83$ and $\mathrm{A} 84$ were alike.

V100 results were obtained at NBS using ASTM D2240. V200 results were obtained at NBS using ASTM D1415.

Four of the 26 particlpants reporting used ASTM D1415 (Wallace)

for the hardness determintation. All others used ASTM D2240

(Type A Durometer).

\begin{tabular}{|c|c|c|c|c|c|c|c|c|}
\hline \multirow[b]{2}{*}{ PROPERTY } & \multirow[b]{2}{*}{ MATERIAL } & \multirow{2}{*}{$\begin{array}{l}\text { LABS } \\
\text { INCL }\end{array}$} & \multirow{2}{*}{$\begin{array}{l}\text { LABS } \\
\text { OMIT }\end{array}$} & \multirow[b]{2}{*}{ GR, MEAN } & \multicolumn{3}{|c|}{ STD DBVIATIENS } & \multirow[b]{2}{*}{ UNITS } \\
\hline & & & & & LABS & SEEBTS & REPL & \\
\hline ARDNBSS & $181=\Delta 82$ & 26 & 0 & 55.26 & 1.72 & .18 & .40 & I RBD \\
\hline & $\triangle 83-A 84$ & 26 & 0 & 56.14 & 1.82 & .20 & $.4 !$ & I RHD \\
\hline
\end{tabular}

PRBCISIGN GF MBTheDS

REPL REPL

PE RCFNT

PREPERTY MATERIAL

GR, MEAN

REPEAT REPRED

UNITS

REPBAT REPRED

HA RDNE 35

AB1-AB2

$\begin{array}{ll}5 & 5 \\ 5 & 5\end{array}$

56.26

1.11

4.77
5.04

IRAD

I RHD

2.0

0.5 


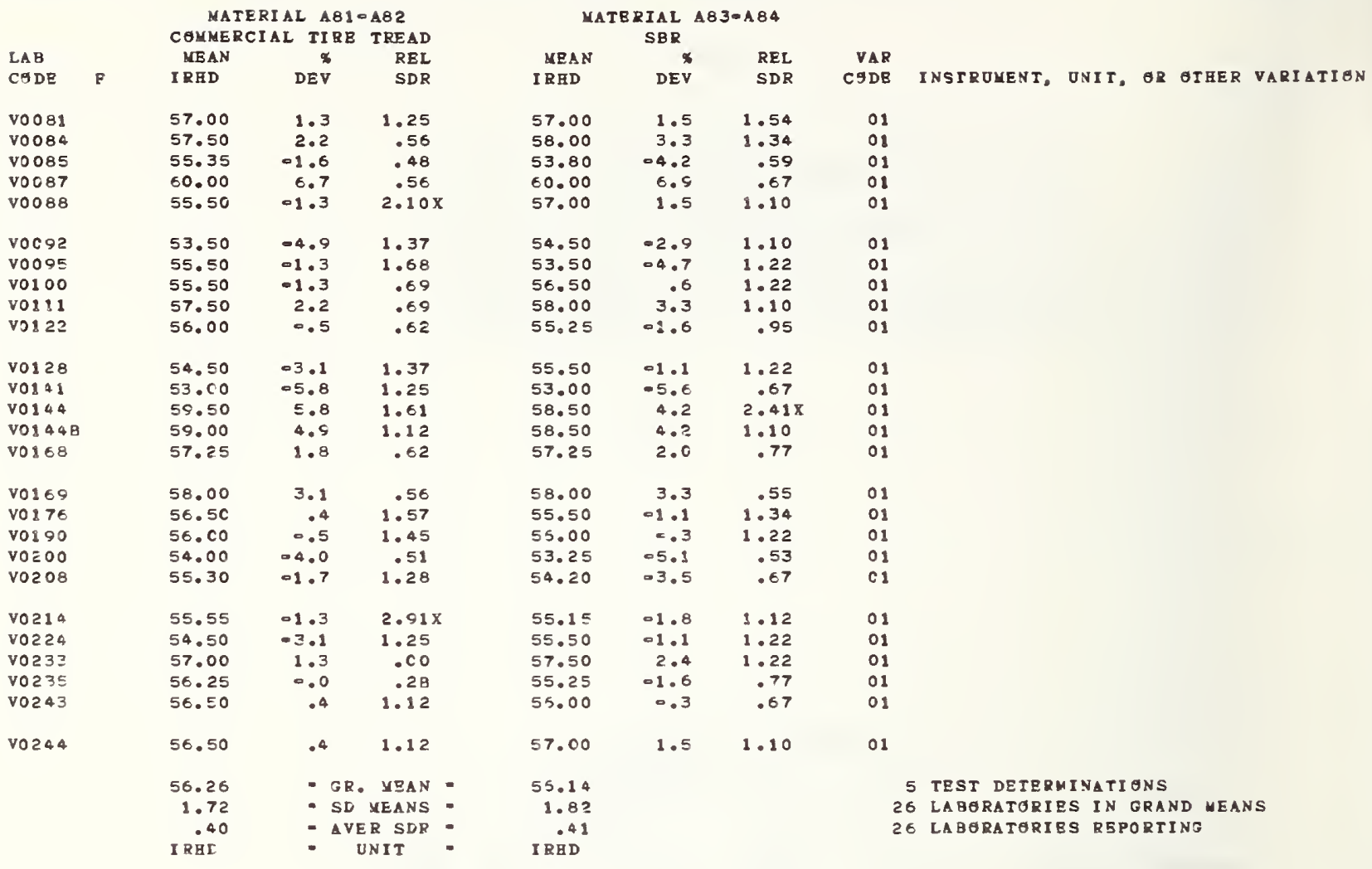


HARDNESS

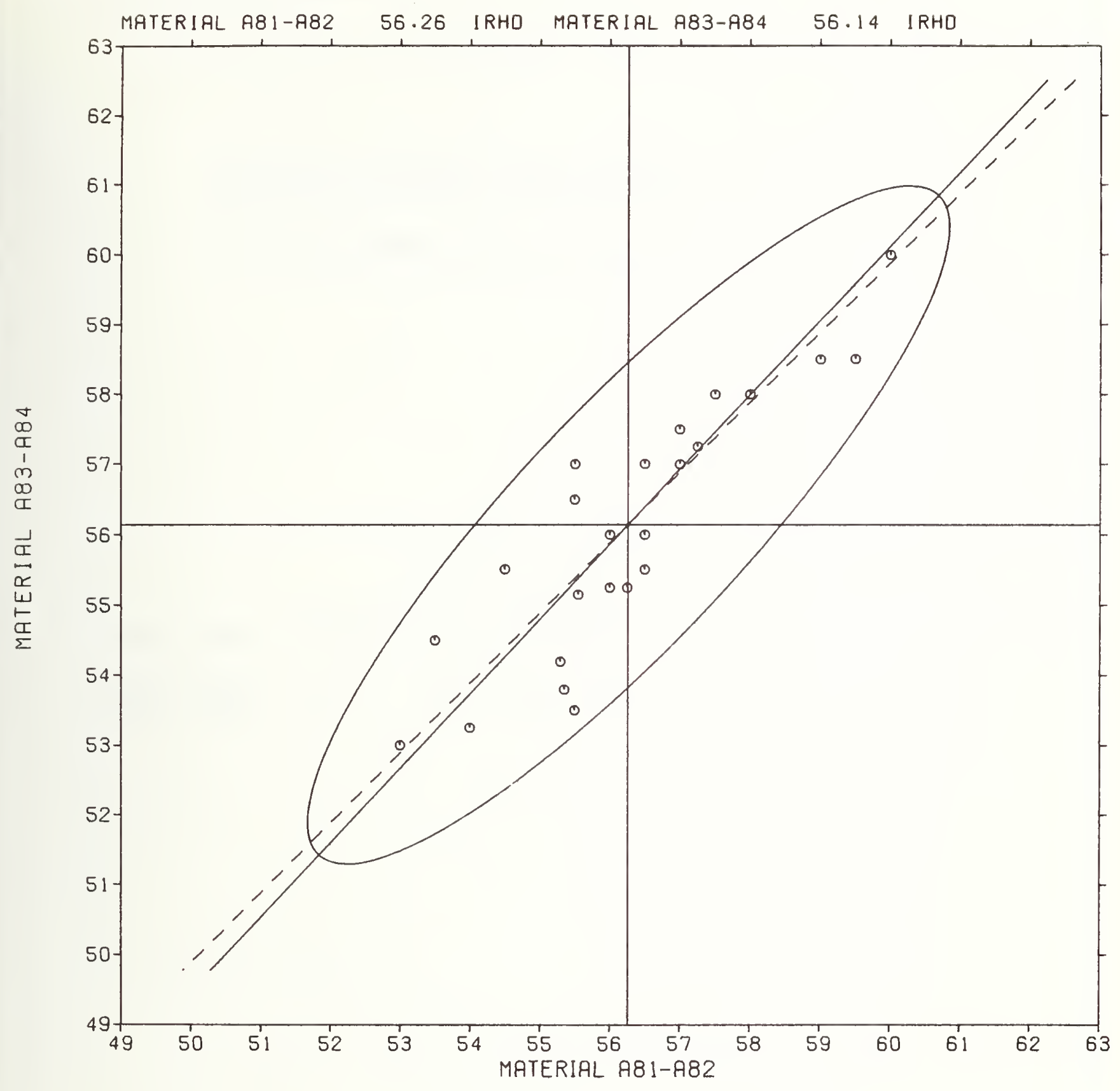




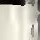


MOONEY VISCOSITY

NOTES

\begin{abstract}
Materials R81 and $R 82$ were the same rubber. Sim1larly, materials $\mathrm{R} 83$ and $\mathrm{R} 84$ were the 8 ame rubber. No sample preparation was required fpr materlals $R 81$ and $R 82$ whereas, m111 massing was required for materials $R 83$ and $R 84$.

V100 results were obtalned at ABS on the manually closed viscometer used for determining the Mooney viscosities of the standard rubbers.
\end{abstract}

SOMKARY OP ANALYSES

\begin{tabular}{|c|c|c|c|c|c|c|c|c|}
\hline \multirow[b]{2}{*}{ PREPERTY } & \multirow[b]{2}{*}{ MATBRIAL } & \multirow{2}{*}{$\begin{array}{l}\text { LABS } \\
\text { INCL }\end{array}$} & LABS & \multicolumn{4}{|c|}{ STD DEVIATIONS } & \multirow[b]{2}{*}{ UNITS } \\
\hline & & & GMIT & GR, MEAN & LABS & SHEETS & REPL & \\
\hline MEENEY & $281-282$ & 40 & 3 & 67.77 & 1.84 & .17 & .35 & ML \\
\hline VIScesITI & $R 83-R 84$ & 40 & 3 & 63.16 & 2.90 & .53 & .45 & ML \\
\hline
\end{tabular}

PRECISIEN GF MBTEGDS

\begin{tabular}{|c|c|c|c|c|c|c|c|c|c|}
\hline \multirow[b]{2}{*}{ PREPERTY } & \multirow[b]{2}{*}{ MATERIAL } & \multirow{2}{*}{$\begin{array}{r}\text { REPL } \\
\text { CRP }\end{array}$} & \multirow{2}{*}{$\begin{array}{l}\text { RBPL } \\
\text { ASTY }\end{array}$} & \multirow[b]{2}{*}{ GR. MEAN } & \multicolumn{2}{|c|}{ ABSELUTB } & \multirow[b]{2}{*}{ UNITS } & \multicolumn{2}{|c|}{ PERCEN I } \\
\hline & & & & & RBPEAT & REPRED & & REPEAT & REPRAD \\
\hline MEONET & $\mathbf{R 8 1 - R 8 2}$ & 3 & 3 & 67.77 & .97 & 5.09 & ML & $2 \cdot 4$ & 7.5 \\
\hline VISCESITY & $R 83-R 8 A$ & 3 & 3 & 63.16 & 1.25 & 8.04 & ML & 2.0 & 12.7 \\
\hline
\end{tabular}


INTERLABGRATGRY PROGRAM ON BVALUATION GP RUBBER

MEANEY VISCESITY - ML

\section{MATBRIAL R83-R84}

MATERIAL REIOR82

\begin{tabular}{|c|c|c|c|c|c|c|c|c|}
\hline \multirow{3}{*}{ LAB } & & \multicolumn{3}{|c|}{ BUTYL RUBBER } & \multicolumn{3}{|c|}{ SBR } & \multirow{2}{*}{ VAR } \\
\hline & & MEAN & $\%$ & REL & MEAN & ๙ & REL & \\
\hline & $\mathbf{P}$ & ML & DBV & SDR & KL & DBV & SDR & CSDB \\
\hline v0077 & & $6 \epsilon .00$ & -2.6 & .72 & 56.50 & -10.5 & .87 & 01 \\
\hline$v 0078$ & $x$ & 65.90 & -2.8 & 1.62 & 50.50 & $=20.0$ & .96 & 01 \\
\hline vo07s & $\mathbf{x}$ & 76.95 & $13 \cdot 5$ & .82 & 68.45 & 8.4 & $4.21 x$ & 01 \\
\hline vooso & $\mathbf{x}$ & 70.45 & 4.0 & 1.37 & 55.95 & -11.4 & $2 \cdot 32$ & 01 \\
\hline vo083 & & 69.25 & 2.2 & .41 & 64.25 & 1.7 & .64 & 01 \\
\hline vo085 & & 67.60 & 0.3 & .16 & 62.10 & -1.7 & $1 \cdot 34$ & 01 \\
\hline vo090 & & 69.15 & 2.0 & .66 & 66.00 & 4.5 & $3.87 x$ & 01 \\
\hline vooge & & 68.00 & $\cdot 3$ & 1.64 & 66.00 & 4.5 & 1.28 & 01 \\
\hline vo095 & & 66.00 & -2.6 & 1.83 & 62.75 &. .7 & 1.43 & 01 \\
\hline Vo1 00 & & 68.25 & .7 & .36 & 62.50 & -1.0 & .00 & 01 \\
\hline $\operatorname{vol} 11$ & & 65.10 & -3.9 & .65 & 59.30 & -6.1 & .34 & 01 \\
\hline v0117 & & 67.00 & -1.1 & 1.12 & 62.25 & -1.4 & 1.40 & 01 \\
\hline v0122 & & 64.75 & -4.5 & 1.12 & 62.00 & -1.8 & .96 & 01 \\
\hline vo128 & & 66.00 & -2.6 & .82 & 63.50 & .5 & .64 & 01 \\
\hline V0144 & & 69.70 & 2.8 & 1.23 & 65.80 & 4.2 & .39 & 01 \\
\hline v0146 & & 70.00 & $3 \cdot 3$ & $2.46 x$ & 67.50 & 6.9 & .32 & 01 \\
\hline VOL 48 & $*$ & 73.10 & 7.9 & .87 & 69.50 & 10.0 & 1.26 & 01 \\
\hline v0149 & & 70.05 & 3.4 & .92 & 64.85 & 2.7 & .65 & 01 \\
\hline v0150 & & 69.90 & 3.1 & .38 & 60.95 & -3.5 & 1.62 & 01 \\
\hline vo1 56 & & $66.0 \mathrm{C}$ & -2.6 & 2.17 & 61.25 & -3.0 & .85 & 01 \\
\hline vo: 66 & & 67.00 & -1.1 & 1.91 & 65.25 & 4.9 & 1.84 & 01 \\
\hline v0169 & & 70.00 & $3 \cdot 3$ & 1.64 & 62.50 & -1.0 & 1.28 & 01 \\
\hline $\operatorname{vol} 77$ & & 64.05 & -5.5 & 1.17 & 63.40 & .4 & .36 & 01 \\
\hline VOI 78 & & $67 \cdot 30$ & .07 & .30 & 62.80 & 0.6 & .50 & $Q_{1}$ \\
\hline v0182 & & 67.75 & 0.0 & .41 & 63.25 & .1 & 1.17 & 01 \\
\hline v0190 & & 68.15 & .6 & .38 & 57.90 & -8.3 & .65 & 01 \\
\hline VO2OE & & 66.25 & -2.2 & 1.23 & 60.00 & -5.0 & 2.25 & 01 \\
\hline V0207 & & 37.40 &. .5 & 1.47 & 64.15 & 1.6 & .89 & 01 \\
\hline vo 208 & & 70.00 & $3 \cdot 3$ & 1.64 & 65.50 & 3.7 & 1.28 & 01 \\
\hline VOE 11 & & 68.00 & $\cdot 3$ & .41 & 62.00 & -1.8 & .00 & 01 \\
\hline vo 213 & & 66.25 & -2.2 & .00 & 60.25 & -4.6 & .32 & 01 \\
\hline vo214 & & 65.25 & 03.7 & 1.50 & 64.00 & $1 \cdot 3$ & $2.58 x$ & 01 \\
\hline vo 217 & & 68.25 & .7 & .41 & 66.00 & 4.5 & .00 & 01 \\
\hline v0218 & & 07.75 & 0.0 & .41 & 63.00 &. .3 & .64 & 01 \\
\hline v0220 & & 69.65 & $2 \cdot 8$ & 1.08 & 66.60 & 5.4 & 1.33 & 01 \\
\hline VOEZ 21 & & 68.60 & 1.2 & .76 & 63.40 & .4 & 1.10 & 01 \\
\hline vo 223 & & 67.75 & .0 & .41 & 69.25 & 9.6 & .64 & 01 \\
\hline$V O=30$ & & 66.35 & -2.1 & 1.80 & 56.50 & -9.9 & .50 & 01 \\
\hline vo2 36 & & 70.00 & 3.3 & .00 & 62.50 & -1.0 & 1.28 & 01 \\
\hline vo 238 & & 66.00 & -2.6 & .82 & 60.50 & -4.2 & $3.58 x$ & 01 \\
\hline vo2 a 4 & & 68.00 & .3 & $3.06 x$ & 64.00 & 1.3 & 1.11 & 01 \\
\hline vo 250 & & 58.00 & $\cdot 3$ & 1.64 & 62.00 & -1.8 & .64 & 01 \\
\hline vo 251 & & 67.30 & 0.7 & 1.57 & 63.35 & .3 & 1.84 & 01 \\
\hline
\end{tabular}

63.16

$\begin{array}{rlr}1.84 & - \text { SD MEANS : } & 2.90 \\ .35 & - \text { AVER SDR - } & .45\end{array}$

ML

- TEST DGTBRMINATIENS

40 LABERATORIBS IN GRAND LBANS

43 LABORATORIGS RBPORTINO 
MOONEY VISCOSITY

MATERIAL R81-R82 67.77 ML MATERIAL R83-R84 63.16 ML

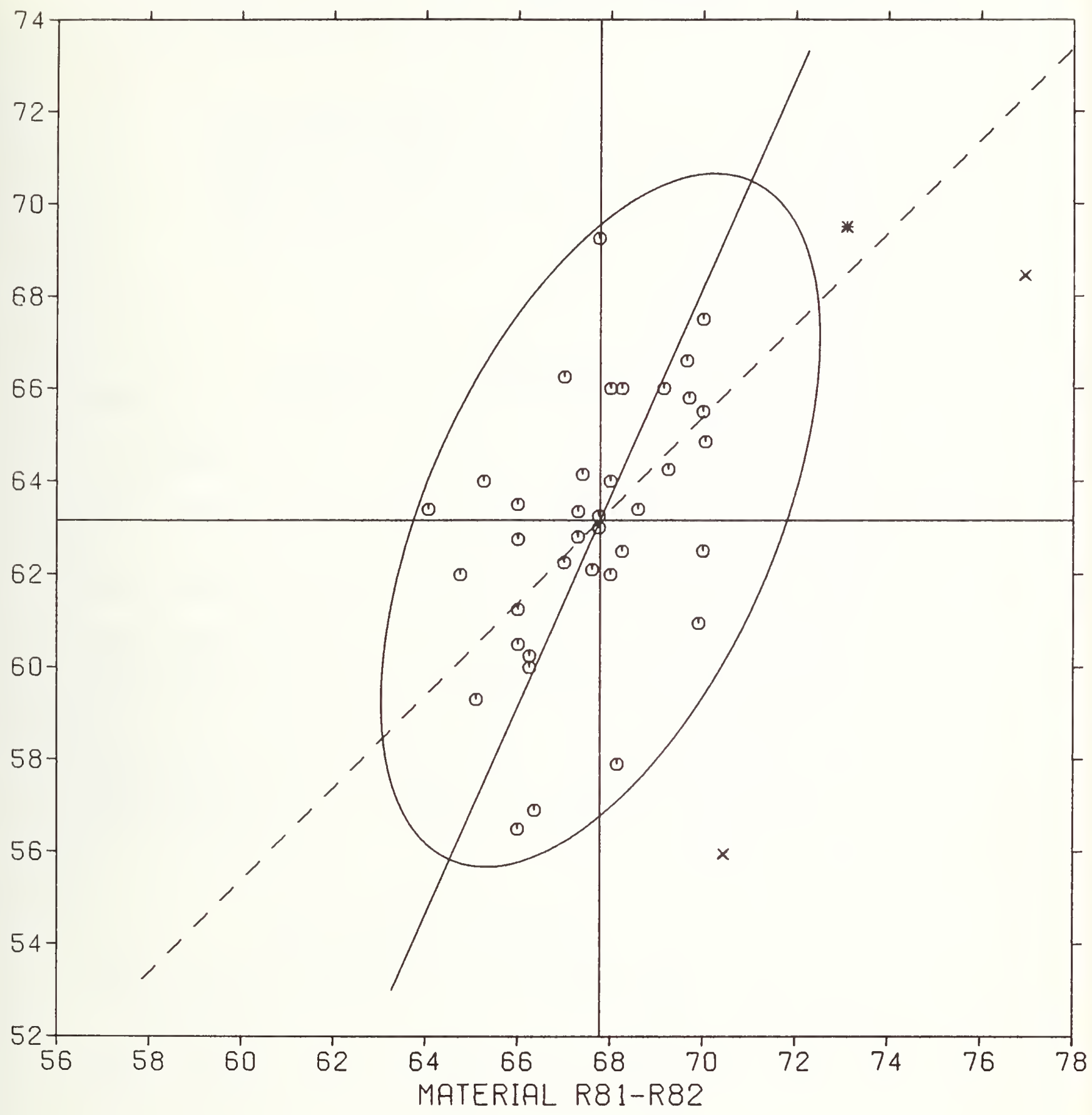


VULCANIZATION CHARACTERISTICS USING OSCILLATING DISK CURE METER

NOTES

Materials W81 and W82 werethe same rubber formulation. Simllarly, materlals W83 and W84 were alike.

V100 results were obtalned at NBS using a Model TM-100 Monsanto Rheometer with a disk osc1llating at $\pm 1^{\circ}$ amplitude and 1.7 hertz frequency.

All particlpants used Monsanto Rheometers operated at one degree amplitude and 1.7 hertz frequency.

\begin{tabular}{|c|c|c|c|c|c|c|c|c|}
\hline \multirow[b]{3}{*}{ PROPERTY } & \multicolumn{8}{|c|}{ SOMMARY OP ANALYSES } \\
\hline & & LABS & LABS & & STD & RVIATIEN & & \\
\hline & MIERIAL & INCL & GUI T & GR. MEAN & LABS & SHBETS & REPL & UNITS \\
\hline ScERCH & $181-\$ 82$ & 37 & 2 & 3.90 & .27 & .03 & .06 & MINUTBS \\
\hline TINE & 183-194 & 37 & 2 & 3.44 & .27 & .02 & .06 & MINOTES \\
\hline CURE IIME & w81-w82 & 36 & 3 & 6.28 & .33 & .03 & .08 & MINUTES \\
\hline (5O\$ ME) & $183-784$ & 36 & 3 & 7.31 & .46 & .05 & .06 & MINUTES \\
\hline CURE TIMB & w81-\$82 & 36 & 3 & 10.53 & .51 & .05 & .13 & MINUTES \\
\hline (90\% Мम) & $183-184$ & 36 & 3 & 14.83 & .96 & .09 & .13 & MINUTES \\
\hline MINIMOM & $\$ 81-782$ & 33 & 6 & 5.11 & .37 & .03 & .07 & PGUND-INCHBS \\
\hline TAROUE & $w 83-w 84$ & 33 & 6 & 6.44 & .41 & .04 & .07 & PEOND-INCHES \\
\hline MINIMUK & w81 $=$ w82 & 33 & 6 & .5778 & .0416 & .0038 & .0075 & NETTEN-MBTERS \\
\hline TOROUR & $183- \pm 84$ & 33 & 6 & .7275 & .0468 & .0046 & .0084 & NE TON-MBTERS \\
\hline MAXIMUM & $m 81-\nabla 82$ & 38 & $\mathbf{1}$ & 24.09 & 1.04 & .08 & .16 & POUNDEINCHES \\
\hline TAROUE & $w 83-\div 84$ & 38 & $i$ & 30.70 & 1.22 & .18 & .09 & POUNDOINCHES \\
\hline MAXIMUK & w81-w82 & 38 & 1 & 2.7217 & .1173 & .0088 & .0177 & NETTON-METBRS \\
\hline TEROUE & $783-784$ & 38 & 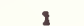 & 3.4684 & .1378 & .0203 & .0105 & HEWION-METBRS \\
\hline
\end{tabular}

PRPCISION OP METHEDS

\begin{tabular}{|c|c|c|c|c|c|c|c|c|c|}
\hline \multirow[b]{2}{*}{ PREPERTY } & \multirow[b]{2}{*}{ MATERIAL } & \multirow{2}{*}{$\begin{array}{r}\text { REPL } \\
\text { CRP }\end{array}$} & \multirow{2}{*}{$\begin{array}{l}\text { REPL } \\
\text { AST }\end{array}$} & \multirow[b]{2}{*}{ GR・MBAN } & \multicolumn{2}{|c|}{ ABSELUTE } & \multirow[b]{2}{*}{ UNITS } & \multicolumn{2}{|c|}{ PRRCENT } \\
\hline & & & & & RBPEAT & RBP RED & & RBPEAT & REPRAD \\
\hline SCORCK & 181-w82 & 3 & 3 & 3.90 & .16 & .75 & MINUTE & 4.2 & 19.1 \\
\hline TIMB & พ $83= \pm 84$ & 3 & 3 & 3.44 & .17 & .75 & KINUTE & 4.9 & 21.9 \\
\hline CORE TIME & w81- -782 & 3 & 3 & 6.28 & .21 & .92 & MINOTB & 3.4 & 10.7 \\
\hline ( $50 \%$ М & $783-784$ & 3 & 3 & 7.31 & .16 & 1.27 & MINOTE & 2.1 & 17.3 \\
\hline CURE TIME & w81-\$82 & 3 & 3 & 10.53 & .37 & 1.42 & MINUTB & 3.5 & 13.4 \\
\hline ( $50 \% \mathrm{NH})$ & $\square 830=84$ & 3 & 3 & 14.83 & .36 & 3.67 & MINUTP & 2.4 & 18.0 \\
\hline MININUM & $w 81-782$ & 3 & 3 & 5.11 & .18 & 1.02 & $L B=I N$. & 3.6 & 19.9 \\
\hline TERQUE & w83-1184 & 3 & 3 & 6.44 & .21 & 1.25 & LB-IN. & 3.2 & 17.8 \\
\hline MINIMUX & $w 81=w 82$ & 3 & 3 & .5778 & .0207 & .1151 & $N=M$ & 3.6 & 19.9 \\
\hline TEROUE & $583=784$ & 3 & 3 & .7275 & .0232 & $.1 \geq 96$ & $N=K$ & $3 \cdot 2$ & 17.9 \\
\hline MAXIMOM & $781=782$ & 3 & 3 & 24.09 & .43 & 2.87 & $L B=I N$ & 1.8 & 18.9 \\
\hline TEROUE & w $3-$ - 84 & 3 & 3 & 30.70 & .26 & 3.38 & $\mathrm{LB}=\mathrm{IN}$ & .8 & 11.0 \\
\hline KAXIMOM & $181-182$ & 3 & 3 & 2.7217 & .0491 & .3248 & $N=M$ & 1.8 & 11.9 \\
\hline TEROUB & w83- & 3 & 3 & 3.4684 & .0292 & .3816 & $N=M$ & .8 & 18.0 \\
\hline
\end{tabular}


INTERLABGRATERY PRGGRAN AN EVALUATIGN OP KUBBER

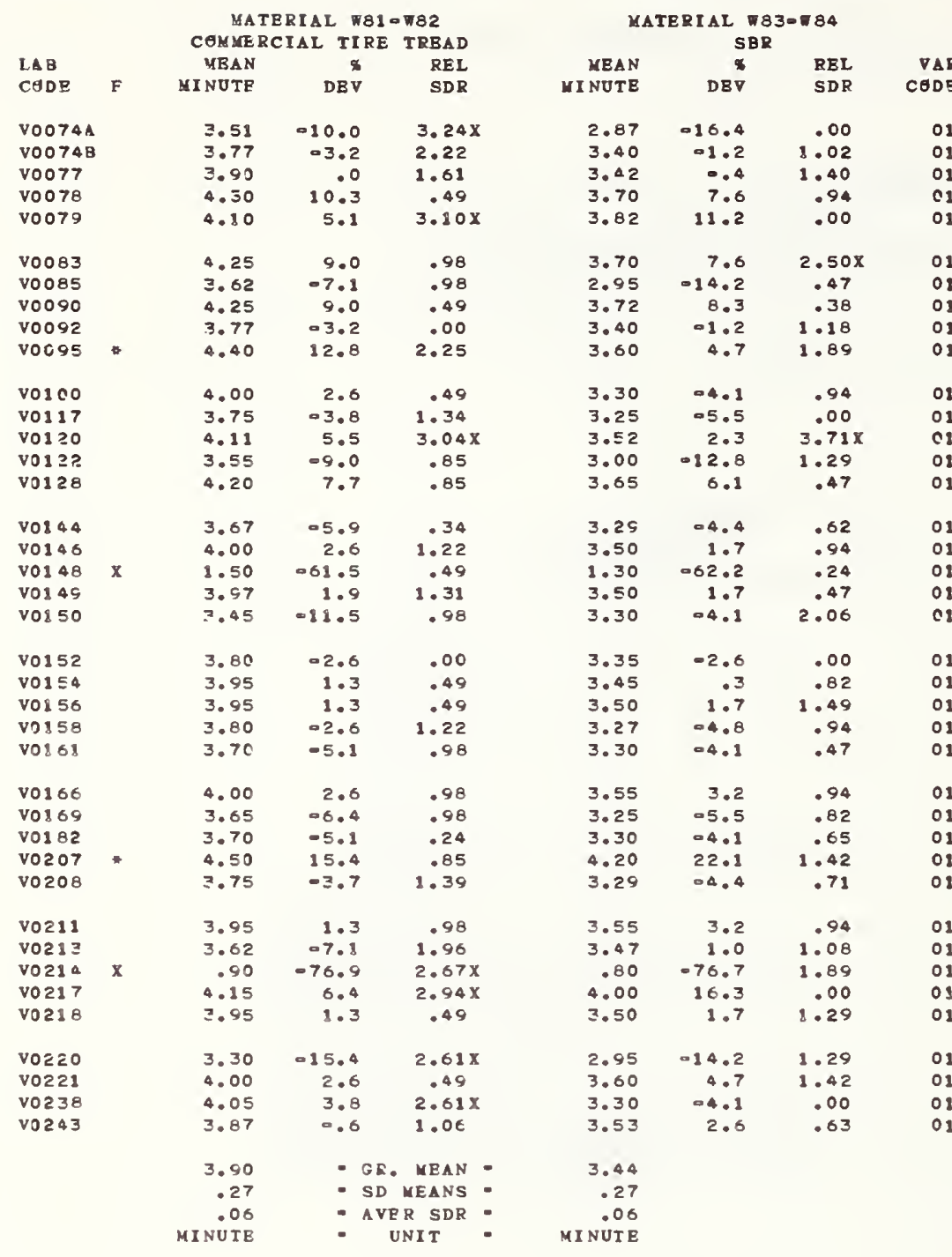

INSTRUMENT, ONIT, OR ATEER VARIATION

$$
01
$$

01

01

01

01

01

01

01

01

C1

01

01

01

c.

01

01

01

01

01

01

08

01

01

01

01

01

01

3 TEST DBTERHINATI ONS

37 LABORATORIES IN GRAND MEANS

39 LABORATERIES RBPORTINO 


\section{SCORCH TIME}

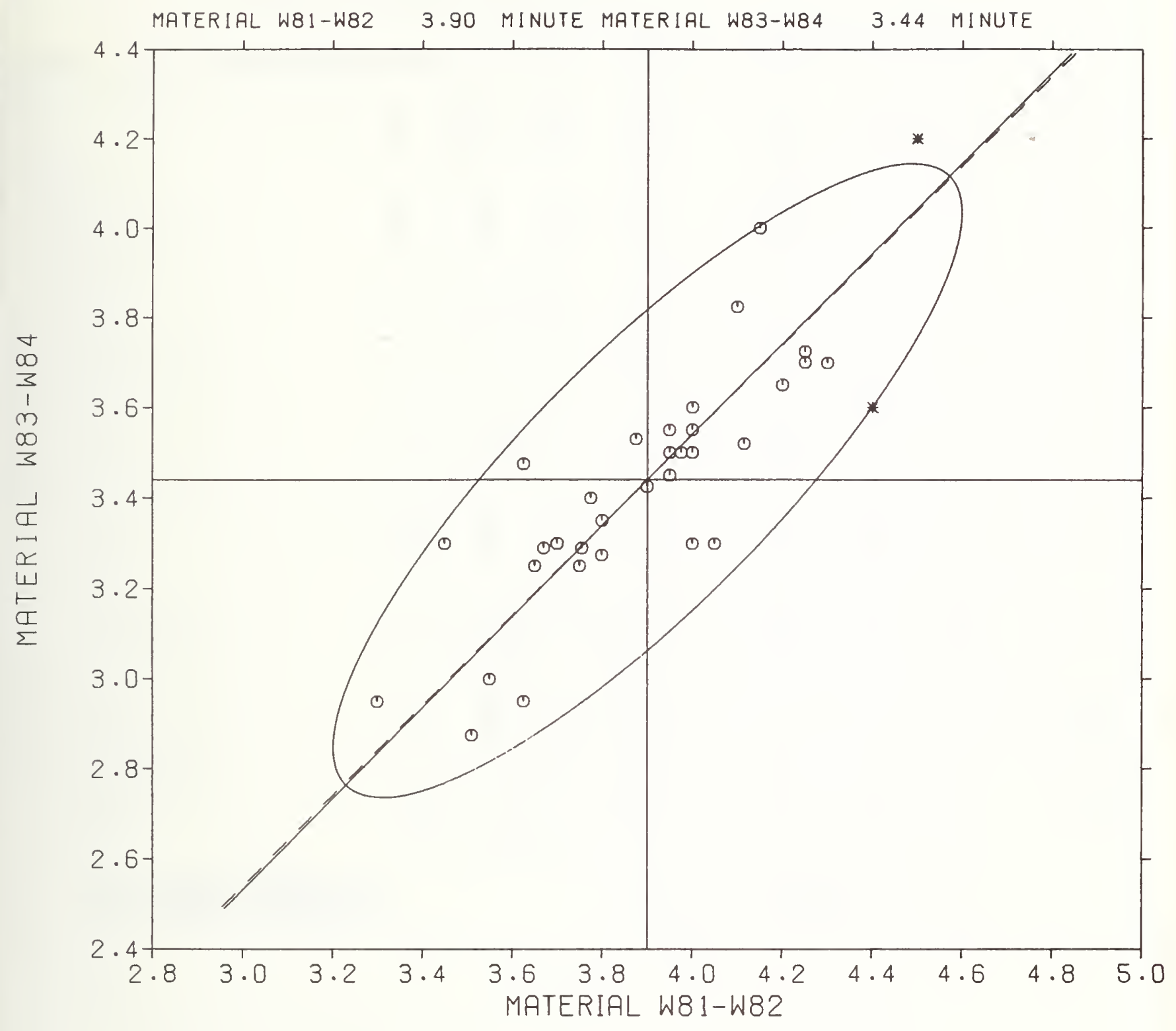




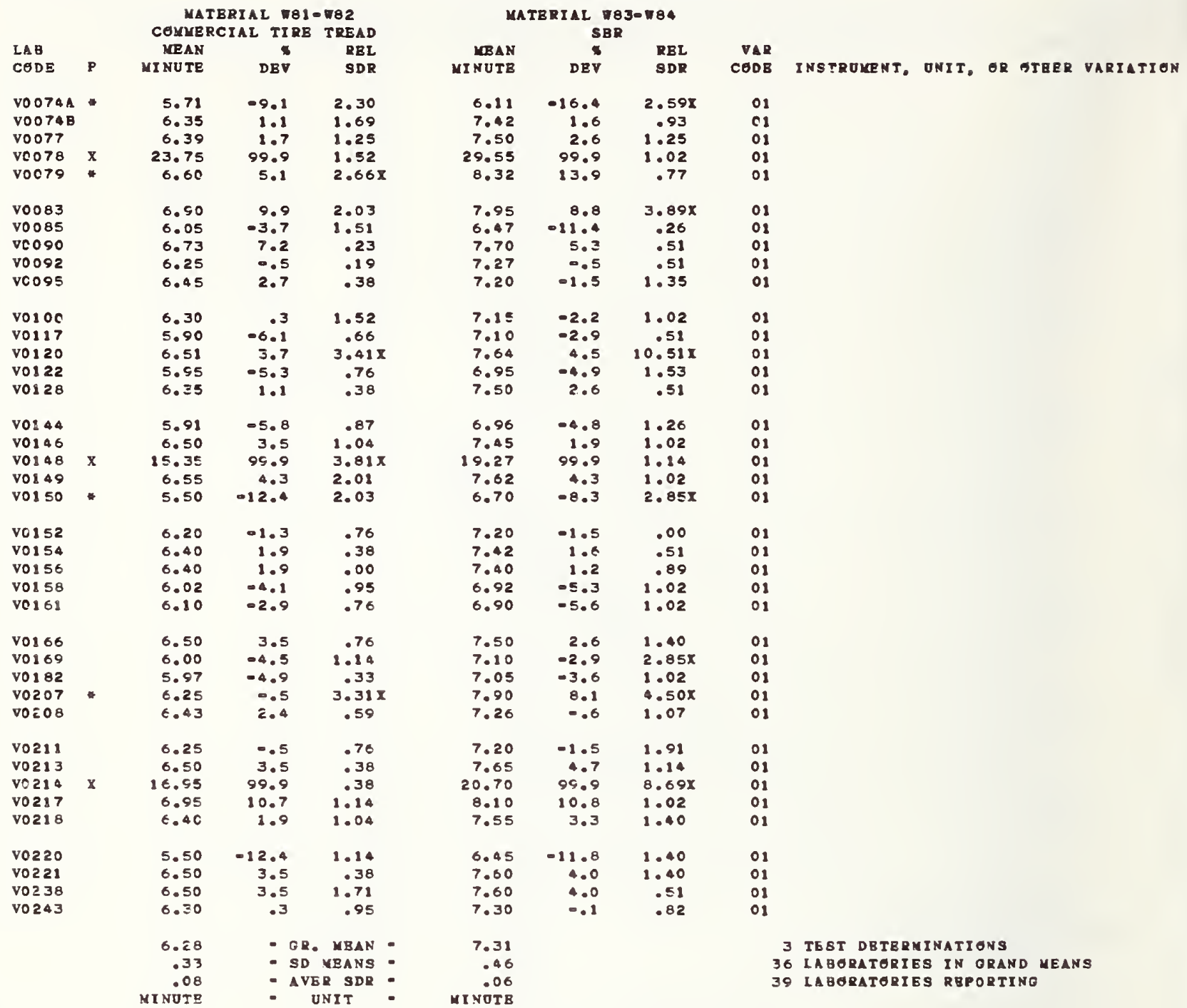


CURE TIME (50\% MH)

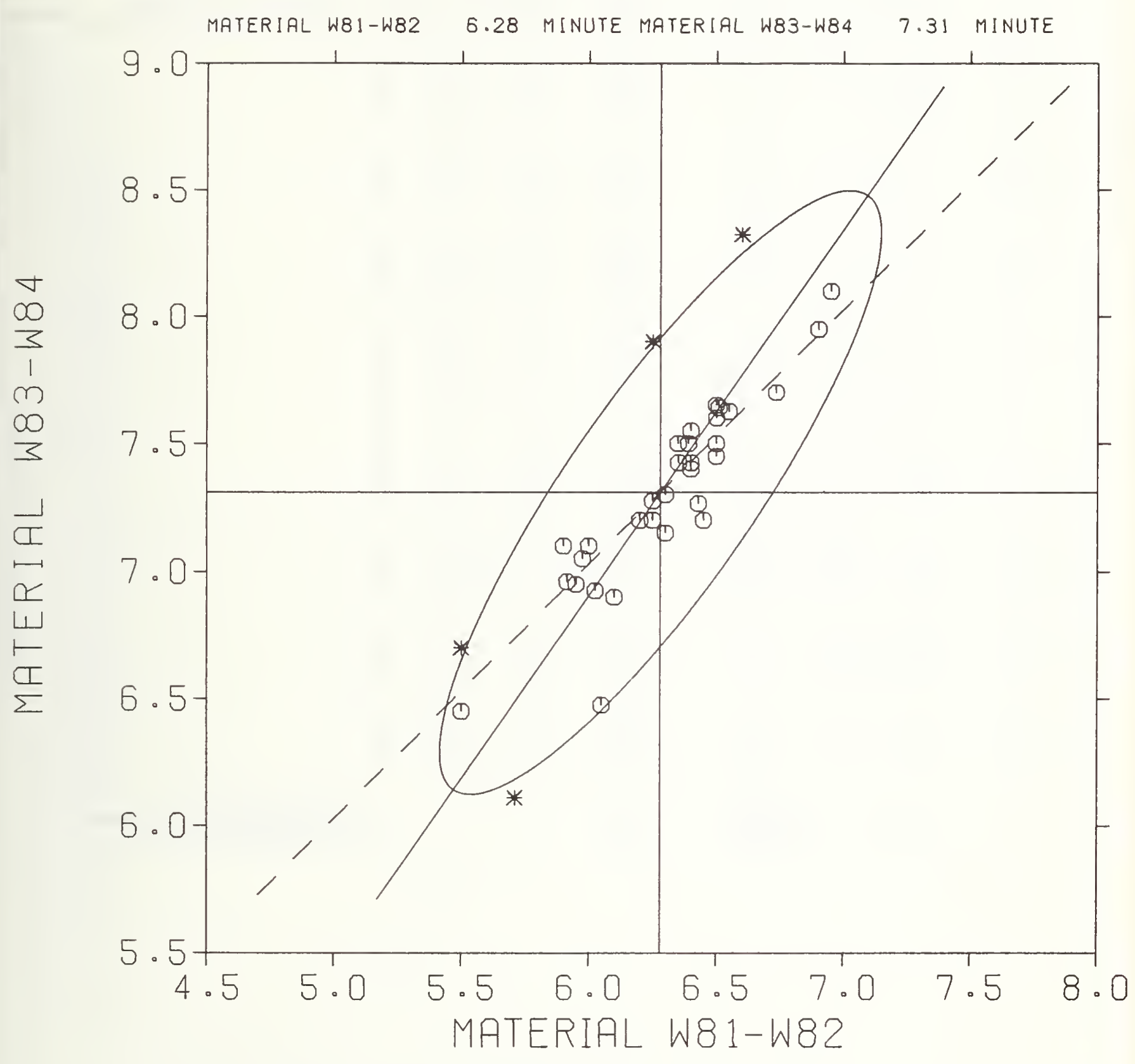




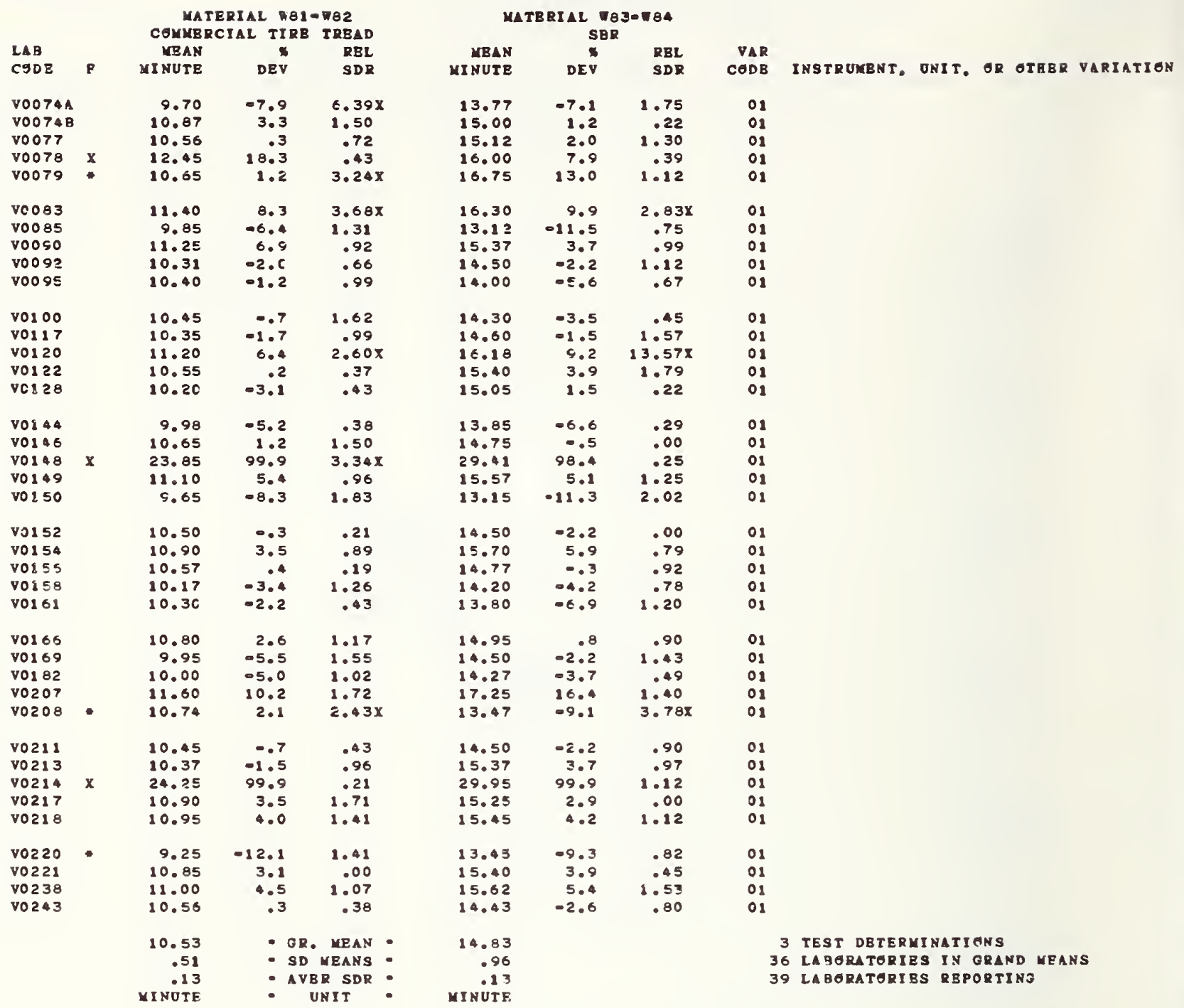


CURE TIME (90\% MH)

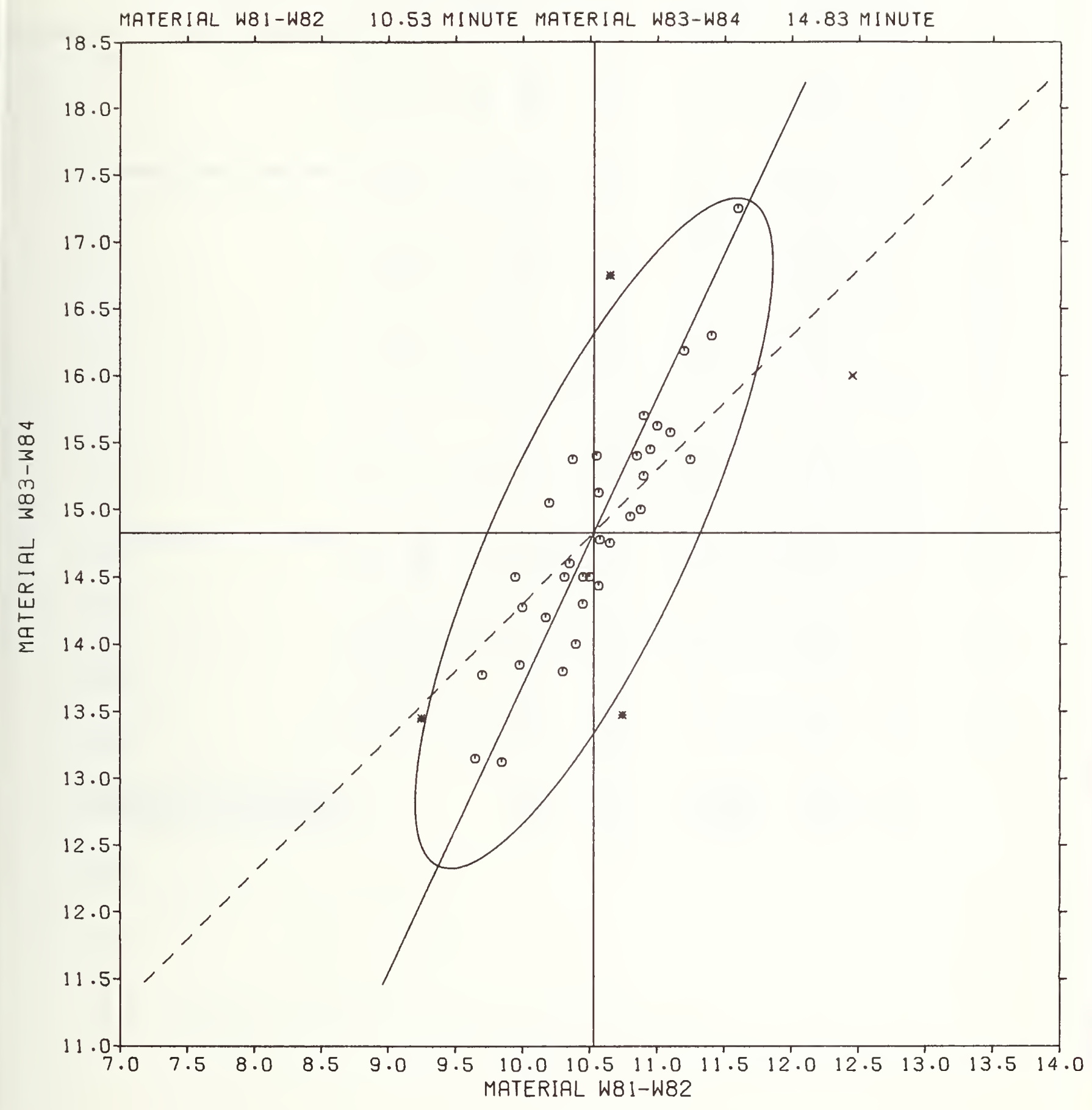




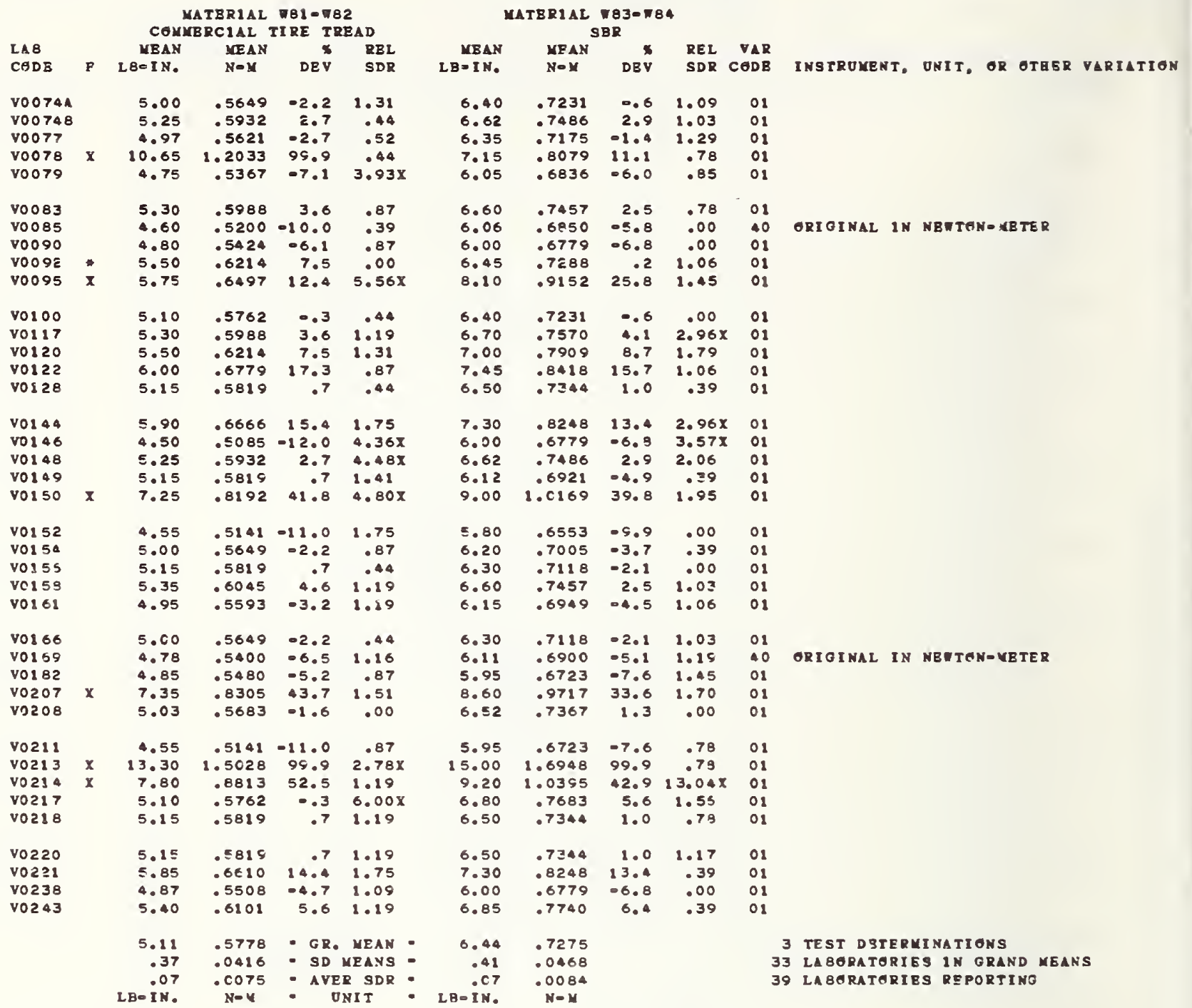


MINIMUM TORQUE

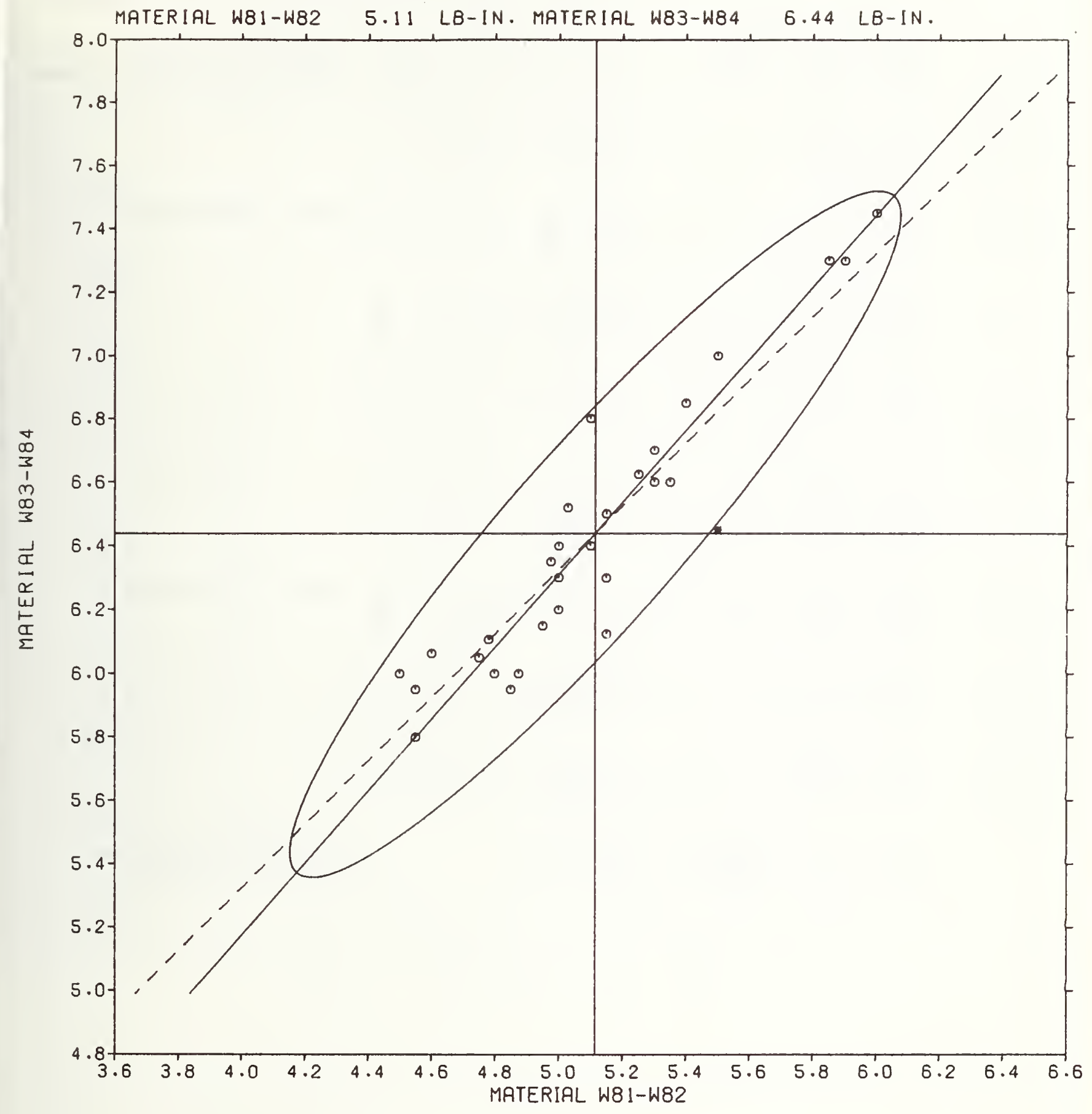




\begin{tabular}{|c|c|c|c|c|c|c|c|c|c|c|c|c|c|}
\hline \multirow{3}{*}{$\begin{array}{l}\text { LA } 8 \\
\operatorname{CoD} B\end{array}$} & & \multirow{2}{*}{\multicolumn{2}{|c|}{ CONKERCIAL }} & & TIRE TREAD & & \multicolumn{4}{|c|}{ MATERIAL T83= "784 } & \multirow{3}{*}{$\begin{array}{r}\text { VAR } \\
\text { CODE }\end{array}$} & \multirow[b]{3}{*}{ INSIRUNENT, ONIT, OR OTBER } & \multirow[b]{3}{*}{ VARTATION } \\
\hline & & MBAN & & 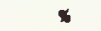 & REL & & MEAN & YEBAN & s & \multirow{2}{*}{$\begin{array}{l}\text { REL } \\
\text { SDR }\end{array}$} & & & \\
\hline & $\mathbf{F}$ & LBoIN. & $N=M$ & $D \Xi V$ & SDR & & $L B=I N$ & $N=M$ & DEV & & & & \\
\hline V0074A & $=$ & 23.72 & 2.6807 & -1.5 & .88 & & 32.45 & $3.6<65$ & 5.7 & 1.00 & 01 & & \\
\hline V0074B & & 24.25 & 2.7400 & .7 & .48 & & 30.70 & 3.4588 & .0 & .94 & 01 & & \\
\hline vo077 & & 23.30 & 2.6327 & $-3 \cdot 3$ & 1.14 & & 29.60 & 3.3445 & -3.6 & 1.13 & 01 & & \\
\hline Vo078 & & 25.25 & 2.8530 & 4.8 & .74 & & 32.05 & 3.6213 & 4.4 & .62 & 01 & & \\
\hline VOC75 & & 23.12 & 2.6129 & -4.0 & $3.31 x$ & & 30.00 & 3.3897 & -2.3 & .00 & 01 & & \\
\hline v0083 & & 25.05 & 2.8304 & 4.0 & 2.05 & & 31.90 & 3.6044 & 3.9 & 1.93 & 01 & & \\
\hline v0085 & & 23.10 & 2.6101 & .4 .2 & .71 & & 29.96 & 3.3851 & -2.4 & .72 & 40 & ORIGINAL IN NBETON-METER & \\
\hline vo090 & & $23 \cdot 45$ & 2.6496 & -2.6 & .32 & & 29.55 & $\equiv .3389$ & -3.7 & .15 & 01 & & \\
\hline V009E & & 25.92 & 2.9293 & 7.6 & .99 & & $32 \cdot 10$ & 3.6270 & 4.6 & .62 & 01 & & \\
\hline vo095 & * & 23.50 & 2.0553 & -2.4 & $4.16 x$ & & 28.00 & 3.1637 & -8.8 & 1.75 & 01 & & \\
\hline Vol oc & & 24.55 & 2.7739 & 1.9 & .18 & & 31.60 & 3.5705 & 2.9 & .00 & 01 & & \\
\hline vo117 & & 24.35 & 2.7513 & 1.1 & 1.21 & & 31.50 & 3.5592 & 2.6 & .93 & 01 & & \\
\hline vo120 & & 25.05 & 2.8304 & 4.0 & 1.00 & & 32.55 & 3.6778 & E. 0 & 2.23 & 01 & & \\
\hline v0122 & & $25 \cdot 20$ & 2.8473 & 4.5 & $2.42 X$ & & 31.90 & 3.5044 & 3.9 & 1.59 & 01 & & \\
\hline Vol $\geq 8$ & & 22.75 & 2.5705 & -5.6 & .82 & & 30.45 & 3.4405 & 0.8 & .31 & 01 & & \\
\hline Vo1 44 & & 25.00 & 2.8248 & 3.8 & .37 & & 31.80 & 3.5931 & 3.6 & $3.09 x$ & 01 & & \\
\hline v0146 & & 22.45 & 2.5356 & -6.8 & .50 & & 28.90 & 3.2654 & -5.9 & .62 & 02 & & \\
\hline VOI 48 & & 25.45 & 2.8756 & 5.7 & 2.18 & & 31.95 & 3.6100 & 4.1 & .31 & 01 & & \\
\hline vo149 & & 23.67 & 2.6750 & -1.7 & 1.54 & & 29.77 & 3.3643 & $=3.0$ & .77 & 01 & & \\
\hline vol 50 & & 25.00 & 2.8248 & 3.8 & 1.84 & & 30.50 & 3.4462 & .06 & $3.09 x$ & 01 & & \\
\hline V015? & & 23.00 & 2.5588 & .4 .5 & .00 & & 29.30 & 3.3106 & .4 .5 & .31 & 01 & & \\
\hline VO1 54 & & 24.00 & 2.7118 &. .4 & .18 & & 30.45 & 3.4405 & .08 & .31 & 01 & & \\
\hline V015E & & 23.20 & 2.6214 & -3.7 & .00 & & 29.40 & 3.3219 & -4.2 & 1.35 & 01 & & \\
\hline vo1 58 & & 24.17 & 2.7315 & .4 & .96 & & 30.80 & 3.4801 & $\cdot 3$ & .54 & 01 & & \\
\hline VOL 61 & & 22.65 & 2.5592 & -6.0 & .81 & & 29.20 & 3.2993 & -4.9 & 1.15 & 02 & & \\
\hline vol 66 & & 23.50 & 2.6553 & - c. 4 & .37 & & 30.50 & 3.4462 & .06 & .62 & 01 & & \\
\hline V01E9 & & 23.81 & 2.6901 & $-1 \cdot 2$ & 1.07 & & 30.14 & 3.4051 & -1.8 & .82 & 40 & GRIGINAL IN NBWTON-MBTER & \\
\hline vol 82 & & 23.85 & 2.6948 & -1.0 & 1.33 & & 31.30 & 3.5366 & 2.0 & 1.07 & 01 & & \\
\hline vo207 & & 26.05 & 2.9434 & 8.1 & 1.44 & & 32.85 & 3.7117 & 7.0 & .85 & 01 & & \\
\hline vo 208 & & 25.33 & 2.8620 & 5.2 & 1.73 & & 31.10 & 3.5140 & $1 \cdot 3$ & $4.51 \times$ & 01 & & \\
\hline vo 211 & & 22.35 & 2.5253 & -7.2 & .58 & & 28.60 & 3.2315 & -6.8 & .31 & 01 & & \\
\hline vo 213 & $x$ & 47.00 & 5.3105 & 95.1 & 2.39 & & 58.75 & 6.6382 & 91.4 & $4.64 x$ & 01 & & \\
\hline vo214 & & 26.05 & 2.9434 & 8.1 & .18 & & 32.15 & 3.6326 & 4.7 & 1.93 & 01 & & \\
\hline VO 617 & & 23.70 & 2.6779 & -1.6 & 2.08 & & 30.30 & 3.4236 & -1.3 & 1.86 & 01 & & \\
\hline Vo218 & & 23.50 & 2.6553 & -2.4 &.$t 6$ & & 30.60 & 3.4575 & .03 & 1.44 & 01 & & \\
\hline VO2 20 & & 23.55 & 2.6609 & -2.2 & $4.222 x$ & & 30.20 & 3.4123 & -1.6 & $2.48 x$ & 01 & & \\
\hline VO2 21 & & 25.00 & 2.8248 & 3.8 & .00 & & 32.10 & 3.6270 & 4.5 & .93 & 01 & & \\
\hline vo2 38 & & 22.87 & 2.5846 & -5.0 & .92 & & 29.25 & 3.3050 & -4.7 & 1.55 & 01 & & \\
\hline vo 243 & & 24.60 & 2.7796 & 2.1 & 1.33 & & 31.00 & 3.5027 & 1.0 & 1.24 & 01 & & \\
\hline & & $<4.09$ & 2.7217 & - $\mathbf{G R}$ & MEAN & - & 30.70 & 3.4684 & & & 3 & TEST DBIERUINATIONS & \\
\hline & & 1.04 & .1173 & - SD $\mathrm{X}$ & IBANS & $\bullet$ & $1.2 \bar{z}$ & .1378 & & & 38 & LABORATORIES IN GRAND NBANS & \\
\hline & & .16 & .0177 & - AVER & SDR & - & .09 & .0105 & & & 39 & LABORATARIES REPORTINO & \\
\hline & & $L B=I N$ & $N=V$ & - $\quad \mathbf{U N}$ & IT & - & LBOIN. & $N=M$ & & & & & \\
\hline
\end{tabular}


MAXIMUM TORQUE

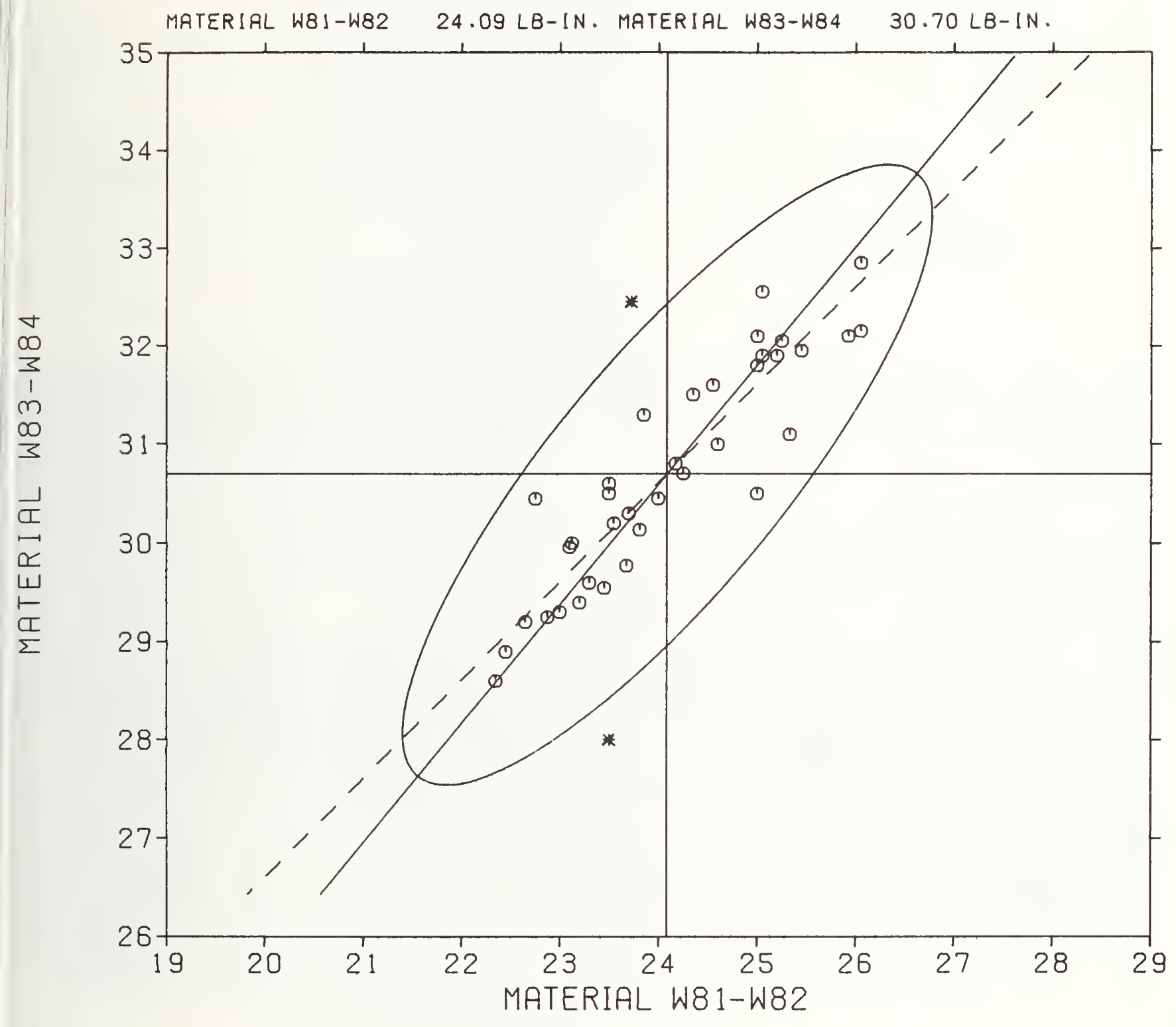


3S-1, 4A (REV, 7-73)

\begin{tabular}{|c|c|c|}
\hline $\begin{array}{l}\text { U.S. DEPT. OF COMM. } \\
\text { BIBLIOGRAPHIC DATA } \\
\text { SHEET }\end{array}$ & $\begin{array}{l}\text { 1. PUIBLIICATION OR RLEPORT NO. } \\
\text { RUBBER CRP } 35\end{array}$ & 3. Recipient's Accession No. \\
\hline \multicolumn{2}{|l|}{ 4. TITLE AND SUBTITLIE } & 5. Publication Date \\
\hline \multicolumn{2}{|c|}{ INTERLABORATORY PROGRAMS FOR RUBBER } & $6 / 12 / 78$ \\
\hline \multicolumn{2}{|c|}{$\begin{array}{l}\text { Analyses: No. } 35 \\
\text { January - March } 1978\end{array}$} & 6. Performing Organization Code \\
\hline \multicolumn{2}{|c|}{$\begin{array}{l}\text { 7. AUTHOR(S) } \\
\text { E. B. Randall, J. Horlick, J. F. Stevenson, G. W. Bullman }\end{array}$} & $\begin{array}{l}\text { 8. Performing Organ. Report No. } \\
\text { NBSIR 78-1339 }\end{array}$ \\
\hline \multirow{2}{*}{\multicolumn{2}{|c|}{$\begin{array}{l}\text { 9. PERFORMING ORGANIZATION NAME AND ADDRESS } \\
\text { NATIONAL BUREAU OF STANDARDS } \\
\text { DEPARTMENT OF COMMERCE } \\
\text { WASHINGTON, D.C. } 20234\end{array}$}} & $\begin{array}{l}\text { 10. Project/Task/Work Unit No. } \\
7825578\end{array}$ \\
\hline & & 11. Contract/Grant No. \\
\hline \multirow{2}{*}{\multicolumn{2}{|c|}{$\begin{array}{l}\text { 12. Sponsoring Organization Name and Complete Address (Street, City, State, ZIP) } \\
\text { Collaborative Testing Services, Inc., } 9241 \text { Wood Glade Drive, } \\
\text { Great Falls, VA } 22066\end{array}$}} & $\begin{array}{l}\text { 13. Type of Report \& Period } \\
\text { Covered } \\
\text { Final }\end{array}$ \\
\hline & & 14. Sponsoring Agency Code \\
\hline
\end{tabular}

16. ABSTRACT (A 200-word or less tactual summary of most significant information. If document includes a significant bibliography or literature survey, mention it here.)

Collaborative Reference Programs provide participating laboratories with the means for checking periodically the level and uniformity of their testing in comparison with that of other participating laboratorles. An important by-product of the programs is the provision of realistic pictures of the state of the testing art. This is one of the periodic reports showing averages for each participant, within and between laboratory variability, and other information for participants and standards committees.

17. KEY WORDS (six to twelve entries; alphabetical order; capitalize only the first letter of the first key word unless a proper name; separated by semicolons)

Collaborative reference program; Laboratory evaluation; Precision;

Reference samples; Rubber; Testing calibration.
18. AVAILABILITY
Unlimited

XX For Official Distribution. Do Not Release to NTIS

Order From Sup. of Doc., U.S. Government Printing Office Washington, D.C. 20402, SD Cat. No. C13

D Order From National Technical Information Service (NTIS) Springfield, Virginia 22151

\begin{tabular}{|l|c|}
\hline $\begin{array}{l}\text { 19. SECURITY CLASS } \\
\text { (THIS REPURT) }\end{array}$ & 21. NO. OF PAGES \\
UNCL ASSIFIED & 36 \\
\hline $\begin{array}{l}\text { 20. SECURITY CLASS } \\
\text { (THIS PAGE) } \\
\text { UNCLASSIFIED }\end{array}$ & 22. Price \\
\hline
\end{tabular}

